\title{
Pointwise Growth and Uniqueness of Positive Solutions \\ for a Class of Sublinear Elliptic Problems where Bifurcation from Infinity Occurs
}

\author{
J. García-Melián, R. Gómez-Reñasco, \\ J. LÓPEZ-Gómez \& J. C. SABINA DE LiS \\ Communicated by P. H. RABINOWITZ
}

\begin{abstract}
In this paper we analyze the uniqueness and the pointwise growth of the positive solutions of a nonlinear elliptic boundary-value problem of general sublinear type with a weight function multiplying the nonlinearity. When this function vanishes on some subdomain, the problem exhibits a bifurcation from infinity. In this case almost nothing is known about the pointwise growth of the positive solutions as the parameter approaches the critical value where the bifurcation from infinity occurs. In this work we show that the positive solutions grow to infinity in the region where the weight function vanishes and that on its support they stabilize to the minimal positive solution of the original equation subject to infinite Dirichlet boundary conditions. This behavior provides us with the uniqueness of the positive solution near the value of the parameter where the bifurcation from infinity occurs. Also, we solve the problem using spectral collocation methods coupled with path-following techniques to show how the main uniqueness result is optimal. Throughout the paper the mathematical analysis aids the numerical study, and the numerical study confirms and illuminates the analysis.
\end{abstract}

\section{Introduction}

In this work we study the pointwise behavior and the uniqueness of positive solutions for the nonlinear boundary-value problem

$$
-\Delta u=\lambda u-a(x)\left(u^{r}+g(x, u)\right) u \quad \text { in } \Omega,\left.\quad u\right|_{\partial \Omega}=0,
$$

where $\Omega \subset \mathbb{R}^{N}, N \geqq 1$, is a bounded domain whose boundary $\partial \Omega$ is of class $C^{1,1}$ (cf. [10]), $\lambda \in \mathbb{R}$ is regarded as a continuation parameter, $r>0$, and $a(x), g(x, u)$ satisfy the assumptions:

(Ha) $a \in C(\bar{\Omega})$ is non-negative, $a \neq 0$. The open set

$$
D:=\{x \in \Omega: a(x)>0\}
$$


satisfies $\bar{D} \subset \Omega$ and possesses a finite number of connected components $D_{j}$, $1 \leqq j \leqq l$, such that $\bar{D}_{i} \cap \bar{D}_{j}=\emptyset$ if $i \neq j$. Each $D_{j}$ is of class $C^{1}$. The open set

$$
\Omega_{0}:=\Omega \backslash \bar{D}
$$

is connected if $N \geqq 2$.

$(\mathrm{H} g)$ The function $g: \bar{\Omega} \times[0,+\infty) \rightarrow \mathbb{R}$, satisfies $\quad g, g_{u}:=\frac{\partial g}{\partial u} \in C(\bar{\Omega} \times$ $[0, \infty) ; \mathbb{R})$ and the growth conditions

$$
\lim _{u \downarrow 0} g(\cdot, u)=0, \quad \lim _{u \uparrow \infty} \frac{g_{u}(\cdot, u)}{u^{r-1}}=0, \quad \text { uniformly in } \bar{\Omega} .
$$

A number of results hold under conditions weaker than $(\mathrm{Hg})$ (cf. Section 2 for details).

Our general setting includes some simple prototype models from population dynamics which have been used as laboratory examples since the late sixties for most of the techniques from nonlinear analysis, e.g., sub- and supersolutions, lo$\mathrm{cal}$ and global bifurcation theory, topological degree and variational methods. For example, in case $g=0$, problem (1.1) becomes

$$
-\Delta u=\lambda u-a(x) u^{r+1} \quad \text { in } \Omega,\left.\quad u\right|_{\partial \Omega}=0,
$$

which is the paradigmatic model in population dynamics (cf. [16]): the logistic problem. In this context $\Omega$ is the region inhabited by the species $u, \lambda$ measures its birth rate and $a(x)$ measures the capacity of $\Omega$ to support the species $u$. Quite surprisingly, the general problem when the species $u$ is free from crowding effects on some subdomain of $\Omega$, i.e., when $a(x)$ vanishes on some subdomain of $\Omega$, has not been tackled until very recently (cf. $[3,17,7]$ and the references therein), although there is a huge amount of literature dealing with the case when $a(x)$ is positive and bounded away from zero.

In order to summarize what it is known for (1.1), (1.2) and give our main results, we need to introduce some notation. Throughout this work, given an open subset $\Omega_{1} \subset \Omega$ with a finite number of components and an elliptic operator $L$ in $\Omega, \sigma_{1}^{\Omega_{1}}[L]$ stands for the principal eigenvalue of $L$ in $\Omega_{1}$ subject to homogeneous Dirichlet boundary conditions on $\partial \Omega_{1}$ (the minimum of the principal eigenvalues of $L$ on each of the components of $\Omega_{1}$ separately). From the relatively recent results of [3, $17,7]$, it readily follows that problem (1.2) possesses a positive solution if and only if $\sigma_{1}^{\Omega}[-\Delta]<\lambda<\sigma_{1}^{\Omega_{0}}[-\Delta]$. Moreover, it is unique, if it exists, and its $L^{\infty}$-norm decays to zero as $\lambda \downarrow \sigma_{1}^{\Omega}[-\Delta]$, and grows to infinity as $\lambda \uparrow \sigma_{1}^{\Omega_{0}}[-\Delta]$. In fact, due to the main theorems of [5] and [18], the point $(\lambda, u)=\left(\sigma_{1}^{\Omega}[-\Delta], 0\right)$ is a bifurcation point for positive solutions of (1.1) from the state $u=0$, and the continuum of positive solution pairs $(\lambda, u)$ emanating from it is unbounded. It turns out that a bifurcation from infinity occurs at the value of the parameter $\lambda=\sigma_{1}^{\Omega_{0}}[-\Delta]$ and that no positive solution is available for $\lambda \geqq \sigma_{1}^{\Omega_{0}}[-\Delta]$ (cf. [13, 9]). In particular, (1.1) possesses at least one positive solution for each $\lambda \in\left(\sigma_{1}^{\Omega}[-\Delta], \sigma_{1}^{\Omega_{0}}[-\Delta]\right)$, 
but now the problem might exhibit more than one positive solution in this range of the parameter $\lambda$ because of the lack of monotonicity of the nonlinearity.

In this paper we ascertain the exact pointwise growth of the positive solutions as $\lambda \uparrow \sigma_{1}^{\Omega_{0}}[-\Delta]$ and analyze the uniqueness of the positive solution. It turns out that the positive solutions grow to infinity uniformly on compact subsets of $\Omega_{0}$ and that they stabilize in $D$, the support of $a(x)$, to the minimal weak solution of the singular boundary-value problem

$$
-\Delta u=\lambda u-a(x)\left(u^{r}+g(x, u)\right) u \quad \text { in } D,\left.\quad u\right|_{\partial D}=\infty .
$$

Singular problems of this type have been previously considered for instance in [11]. Our theory provides us with a broad class of realistic models where these singular problems arise in a natural way. Also, we show that when $a(x)$ is sufficiently smooth on $\partial D$, then the divergence to infinity is uniform on any compact subset of $\Omega_{0} \cup \partial D$. The problem of analyzing the limiting behavior on the interphase $\partial D$ for general non-smooth weights seems very difficult to handle, and remains open.

That the positive solutions of (1.1) grow to infinity in $\Omega_{0}$ as $\lambda \uparrow \sigma_{1}^{\Omega_{0}}[-\Delta]$ while they stabilize on $D$ is rather striking as it entails that a global interior Harnack inequality uniform in compact subintervals of the parameter $\lambda$ is not available for (1.1), in strong contrast with the case when $a(x)$ is positive and bounded away from zero, where the theory developed in [8] shows the validity of a global interior Harnack inequality uniform on compact subintervals of $\lambda>\sigma_{1}^{\Omega}[-\Delta]$.

As to the uniqueness of the positive solution, our main result shows that if either the thickness of the support of $a(x)$ or the amplitude of $a(x)$ is sufficiently small, then (1.1) possesses a unique positive solution for $\lambda$ in a left-neighborhood of $\sigma_{1}^{\Omega_{0}}[-\Delta]$. Also, we complement the analysis with some numerical computations illustrating the pointwise growth of the positive solutions of (1.1) as $\lambda \uparrow \sigma_{1}^{\Omega_{0}}[-\Delta]$ and showing that our uniqueness result is optimal in the sense that if the amplitude of $a(x)$ grows, then the set of $\lambda$ 's for which (1.1) possesses a unique positive solution becomes smaller.

An outline of this paper is as follows: In Section 2 we study the existence of positive solutions for (1.2) and (1.1). Although most of these results are known, we state them in our general setting for further use, and sometimes we give new shorter proofs of them. Also, we solve (1.1) using spectral collocation methods coupled with path-following techniques. This gives high accuracy with low computational work. Our numerical computations predict the pointwise growth of the positive solutions as $\lambda$ approaches $\sigma_{1}^{\Omega_{0}}[-\Delta]$. In Section 3 we analyze how these solutions grow to infinity on $\Omega_{0}$ and $\partial D$. In Section 4 we first show the uniqueness result referred to above and then give another uniqueness result of a different nature, which will be used in Section 6 to show the stabilization of the positive solutions in $D$. In Section 5 we use the numerical computations introduced in Section 2 to discuss the optimality of the main uniqueness result. Finally, in Section 6 we show the stabilization of the positive solutions in $D$ towards the minimal positive solution of problem (1.3). 


\section{On the Existence of Positive Solutions}

The existence results are valid under a condition weaker than $(\mathrm{Hg})$. Notice that $(\mathrm{H} g)$ implies that

$$
\lim _{u \uparrow \infty} \frac{g(\cdot, u)}{u^{r}}=0 \quad \text { uniformly in } \bar{\Omega}
$$

while from the regularity assumption on $g$ it follows that $|g(x, u)-g(x, v)| /|u-v|$ remains bounded on bounded sets of $\bar{\Omega} \times[0, \infty)$. For the rest of this section and Section 3 the following weaker hypotheses will be enough:

(Hgw) $g \in C(\bar{\Omega} \times[0, \infty))$ is locally Lipschitz continuous in $\bar{\Omega} \times[0, \infty)$ with respect to $u$ and

$$
\lim _{u \downarrow 0} g(\cdot, u)=0, \quad \lim _{u \uparrow \infty} \frac{g(\cdot, u)}{u^{r}}=0, \quad \text { uniformly in } \bar{\Omega} .
$$

Definition 2.1. A function $u: \bar{\Omega} \rightarrow[0, \infty)$ is said to be a solution of (1.1) if $u \in L^{\infty}(\Omega) \cap W^{1, p_{0}}(\Omega)$ for some $p_{0}>1$ and (1.1) is satisfied in the weak sense.

Lemma 2.2. If $u$ is a solution of (1.1), then $u \in W^{2, p}(\Omega) \cap W_{0}^{1, p}(\Omega)$ for all $p>1$ and hence, $u \in C^{1, v}(\bar{\Omega})$ for all $0<v<1$. Moreover, $u$ is a.e. in $\Omega$ twice continuously differentiable. In other words, $u$ is a strong solution of (1.1).

Proof. The $L^{p}$-estimates of Agmon, Douglis \& Nirenberg (cf. [10, Chapter IX] ) show that $u \in W^{2, p}(\Omega) \cap W_{0}^{1, p}(\Omega)$ if $p>1$. The remaining assertions follow from the embedding $W^{2, p}(\Omega) \subset C^{2-N / p}(\bar{\Omega}), p>N$, and Theorem VIII.1 of [19]. $\square$

Remark 2.3. If $u$ is a positive solution of (1.1), then the maximum principle implies that $u(x)>0$ for all $x \in \Omega$ and that $\frac{\partial u}{\partial n}(x)<0$ for all $x \in \partial \Omega$, where $n$ is the outward unit normal to $\Omega$ at $x$ (cf. [10, Chapter IX], and [20]), i.e., $u$ lies in the interior of the cone of positive functions of $C_{0}^{1}(\bar{\Omega})$.

If $u \rightarrow u^{r}+g(\cdot, u)$ is increasing, then the following result holds.

Theorem 2.4. Assume that a $(x)$ satisfies $(\mathrm{H} a), g \in C(\bar{\Omega} \times[0, \infty))$ is locally Lipschitz continuous with respect to $u, g(\cdot, 0)=0$, the mapping $u \rightarrow u^{r}+g(\cdot, u)$ is increasing, and

$$
\lim _{u \uparrow \infty}\left(u^{r}+g(\cdot, u)\right)=\infty \quad \text { uniformly in } \bar{\Omega} .
$$

Then (i) Problem (1.1) possesses a positive solution if and only if

$$
\sigma_{1}^{\Omega}[-\Delta]<\lambda<\sigma_{1}^{\Omega_{0}}[-\Delta]
$$

Moreover, it is unique if it exists.

(ii) Let (2.3) hold and let $\theta_{[\lambda, a, g]}$ denote the unique positive solution of (1.1). Then,

$$
\lim _{\lambda \downarrow \sigma_{1}^{\Omega}[-\Delta]}\left\|\theta_{[\lambda, a, g]}\right\|_{L^{\infty}(\Omega)}=0, \quad \lim _{\lambda \uparrow \sigma_{1}^{\Omega_{0}}[-\Delta]}\left\|\theta_{[\lambda, a, g]}\right\|_{L^{\infty}(\Omega)}=\infty .
$$


(iii) The mapping $\lambda \rightarrow \theta_{[\lambda, a, g]}$ is pointwise increasing. If it is regarded as a mapping from the interval (2.3) into $C^{1, v}(\bar{\Omega}), 0<v<1$, then it is continuous. In fact, if $g_{u} \in C(\bar{\Omega} \times[0, \infty))$, then this mapping is differentiable and $\frac{\partial \theta_{[\lambda, a, g]}}{\partial \lambda} \in$ $W^{2, p}(\Omega) \cap W_{0}^{1, p}(\Omega)$ for all $p>1$.

The existence and the uniqueness were found in [3] by using variational methods. The corresponding result in the context of problem (1.2) was independently found in [17] by means of global continuation methods. The second half of (iii) was found in [7], where the method of sub- and supersolutions was used to show that Theorem 2.4 is also true for general second-order elliptic operators not necessarily selfadjoint and that in fact $\theta_{[\lambda, a, g]}$ is a global attractor for all positive solutions of the associated parabolic problem. It should be remarked that the regularity of $\partial D$ can be weakened to the requirement that $\partial D$ has the segment property (cf. [2]) with respect to $\Omega_{0}$. It remains to show the first half of (iii).

Proof. To show that $\theta_{[\lambda, a, g]}$ increases in $\lambda$, note that if $\lambda_{2}>\lambda_{1}$, then $\theta_{\left[\lambda_{2}, a, g\right]}$ is a positive strict supersolution of (1.1) with $\lambda=\lambda_{1}$. Hence, by the monotonicity of the nonlinearity, the strong maximum principle implies that $\theta_{\left[\lambda_{2}, a, g\right]} \gg \theta_{\left[\lambda_{1}, a, g\right]}$ in the sense that $\theta_{\left[\lambda_{2}, a, g\right]}-\theta_{\left[\lambda_{1}, a, g\right]}$ lies in the interior of the cone of positive functions of $C_{0}^{1}(\bar{\Omega})$. For the continuity of $\theta_{[\lambda, a, g]}$ in $\lambda$ pick $\sigma_{1}^{\Omega}[-\Delta]<\hat{\lambda}<\sigma_{1}^{\Omega_{0}}[-\Delta]$ and a sequence $\lambda_{n} \in\left(\sigma_{1}^{\Omega}[-\Delta], \sigma_{1}^{\Omega_{0}}[-\Delta]\right), n \geqq 1$ such that $\lim _{n \rightarrow \infty} \lambda_{n}=\hat{\lambda}$. Set $\lambda_{\infty}=\sup _{n \geqq 1} \lambda_{n}$. Then, for each $n \geqq 1$, we have $\theta_{\left[\lambda_{n}, a, g\right]} \leqq \theta_{\left[\lambda_{\infty}, a, g\right]}$ and hence $\left\|\theta_{\left[\lambda_{n}, a, g\right]}\right\|_{L^{\infty}(\Omega)} \leqq C, n \geqq 1$, for some constant $C$. This provides us with uniform bounds for $\left\|\Delta \theta_{\left[\lambda_{n}, a, g\right]}\right\|_{L^{\infty}(\Omega)}$ and hence, by the elliptic $L^{p}$ estimates of AGMON, Douglis \& NIRENBERG and by MORREY's Theorem we get uniform bounds for $\left\|\theta_{\left[\lambda_{n}, a, g\right]}\right\|_{C^{1, v}(\bar{\Omega})}, 0<v<1$. Fix $v \in(0,1)$ and pick $\hat{v} \in(0, v)$. Then, there exists a subsequence of $\theta_{\left[\lambda_{n}, a, g\right]}$, relabeled by $n$, such that

$$
\lim _{n \rightarrow \infty}\left\|\theta_{\left[\lambda_{n}, a, g\right]}-u\right\|_{C^{1, \hat{v}}(\bar{\Omega})}=0
$$

for some $u \in C^{1, \hat{v}}(\bar{\Omega})$. Necessarily $u$ is a non-negative solution of (1.1) with $\lambda=\hat{\lambda}$. Moreover, $u \neq 0$, since $\lambda=\sigma_{1}^{\Omega}[-\Delta]$ is the unique value of $\lambda$ for which bifurcation to positive solutions from the state $(\lambda, u)=(\lambda, 0)$ may occur. Therefore, by the uniqueness of the positive solution, $u=\theta_{[\hat{\lambda}, a, g]}$. Since this argument is valid along any subsequence, the proof is completed.

To study the solution curves of (1.1) and the behavior of the positive solutions along them we have carried out some numerical calculations. We solve (1.1) using spectral collocation methods coupled with path-following techniques. This gives high accuracy with low computational work. In all our numerical calculations we have used trigonometric modes and the collocation points have been taken to be equidistant, with the number of modes equal to the number of collocation points. The details of our numerical scheme may be found in [14] and the references therein. We refer the interested reader to [14]. Figure 2.1 shows a typical bifurcation diagram of positive solutions for (1.1) under the assumptions of Theorem 2.4. The numerical 

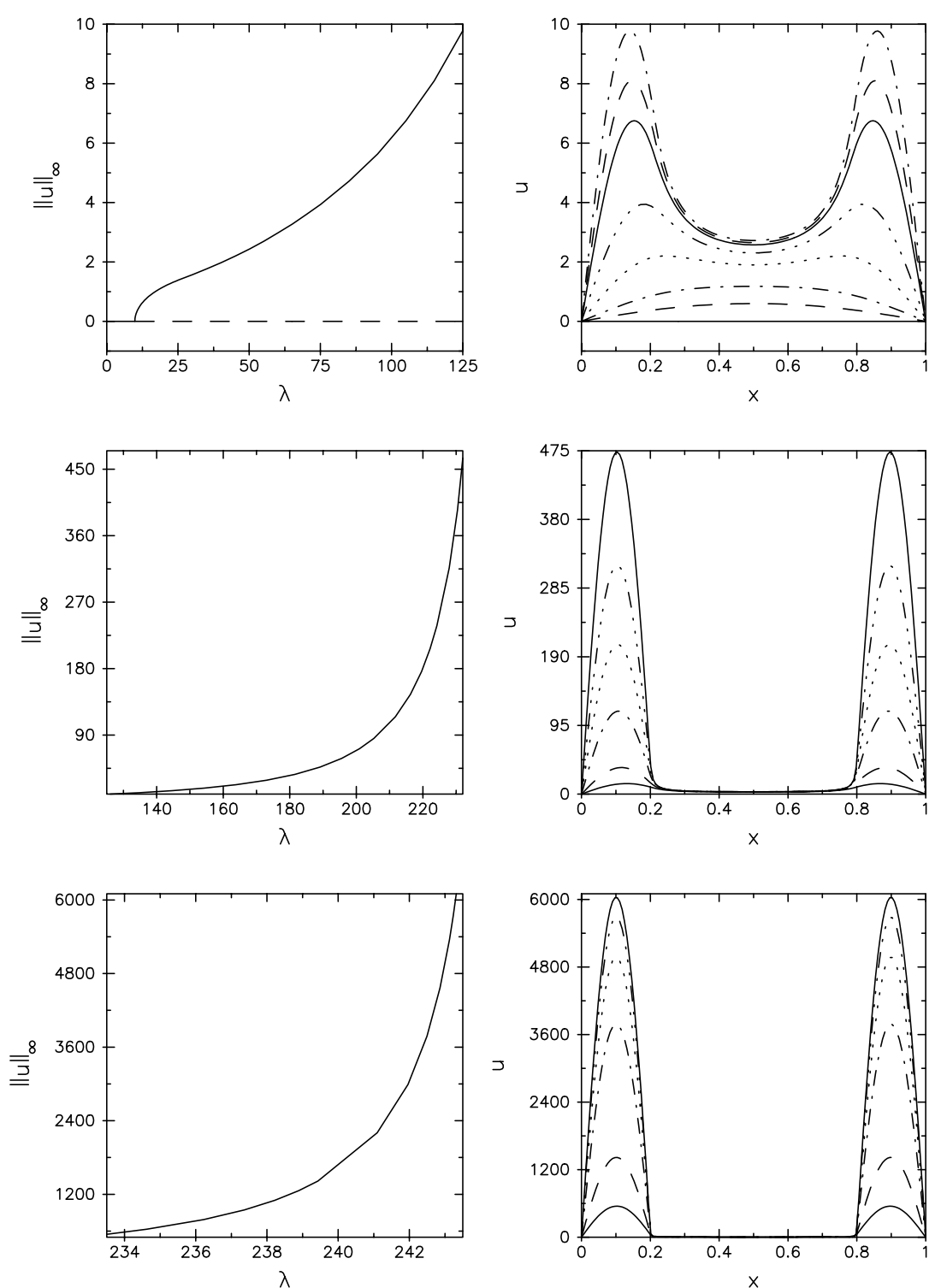

Fig. 2.1. Bifurcation diagram under the assumptions of Theorem 2.4.

calculations were carried out for the choices

$$
\Omega=(0,1), r=4, g(x, u)=11 u^{2}, a(x)=\max \left\{0, \frac{4}{9}\left(-25 x^{2}+25 x-4\right)\right\} .
$$

Note that $a(x)=0$ if and only if $x \in[0,0.2] \cup[0.8,1]$ and that $a(x)>0$ if $x \in(0.2,0.8)$. Thus, in this example, $\Omega_{0}=(0,0.2) \cup(0.8,1)$ and hence

$$
\sigma_{1}^{\Omega}[-\Delta]=\pi^{2} \simeq 9.8696, \quad \sigma_{1}^{\Omega_{0}}[-\Delta]=(5 \pi)^{2} \simeq 246.7401 .
$$


In Figure 2.1 we have divided the $\left(\lambda,\|\cdot\|_{\infty}\right)$-diagram of non-negative solutions into three pieces, those in the first column, corresponding to a different range of values of $\lambda$. These variation ranges are the intervals $(0,125),(125,233)$ and $(233,243.5)$, respectively (from the top to the bottom). Continuous lines represent stable solutions and dashed lines unstable solutions, each point on these curves representing a nonnegative solution of (1.1). The first diagram represents the trivial state $u=0$ together with the curve of positive solutions emanating from it at $\pi^{2}$, where the trivial state loses stability. Since this bifurcation is supercritical, by the principle of exchange of stability (cf. [6]) the positive solutions are stable for $\lambda \simeq \pi^{2}$. The main theorem of [7] shows that in fact any positive solution is stable. As shown in Figure 2.1, this agrees with our numerical calculations.

Each of the figures on the second column of Figure 2.1 shows the profiles of some of the solutions along the corresponding piece of the diagram on its left. The figure on the first row shows the positive solutions for each of the following values of $\lambda: 9.8696,12.2517,20.2219,45.1851,75.1397,105.0561,115.0055$ and 124.9319, respectively. Of course, the solutions grow as $\lambda$ increases (cf. Theorem 2.4 (iii)). The figure on the second row shows the positive solutions for $\lambda=144.6433,181.5242$, $211.7337,222.1816,227.9162$ and 232.1828 , and the figure on the third row shows the positive solutions for $\lambda=233.5460,239.4319,242.4959,243.0061,243.2158$ and 243.3033. Note how the $L^{\infty}(\Omega)$-norms of the positive solutions grow as $\lambda$ increases.

To carry out the numerical computations we have used 63 modes to complete the calculation of the solution corresponding to $\lambda$ up to $\lambda=105.0561$, and for larger values of $\lambda$ we have used modes 143 in order to increase the accuracy near the bifurcation point from infinity. A lower number of modes makes impossible the calculation of the positive solutions for values of $\lambda$ close to $\sigma_{1}^{\Omega_{0}}$, as we shall soon show. The numerical Jacobian of the linearization of the discrete approximation of (1.1) at the computed solution decreases along the curve, decaying to zero as $\lambda$ approaches the value 243.3718 . Then, it increases, while the computed solution decreases. Thanks to Theorem 2.4 (iii) we already now that this is impossible. Therefore, we must stop the computations here and propose the following numerical value for the point where bifurcation from infinity occurs:

$$
\text { numerical } \sigma_{1}^{\Omega_{0}} \simeq 243.3718 \text {. }
$$

At first glance this value is far away from the value given by Theorem 2.4, namely, $\sigma_{1}^{\Omega_{0}} \simeq 246.7401$. Fortunately, this difference can be explained from the fact that in the numerical calculations we are reducing the support of $a(x)$ to the interval $(0.2013,0.7987)$, because 0.2013 and 0.8 are the first collocation points after 0.2 and 0.7987 , respectively, and therefore the numerical $\sigma_{1}^{\Omega_{0}}$ should approximate $(\pi / 0.2013)^{2} \simeq 243.5635$ rather than 246.7401 . Of course, for our purposes here 243.3718 is an excellent numerical approximation. This explains as well why a high number of modes might be necessary to get a reasonable approximation when spatially varying coefficients arise in the formulation of the model, in strong contrast with the case of constant coefficients where in general a low number of modes are sufficient to get good numerical approximations even when dealing with systems (cf. [14] and the references therein). 
A striking feature is that the positive solution grows to infinity in $\Omega_{0}$ while it stabilizes on $D$, the support of $a(x)$, as $\lambda$ approaches 243.3718. This shows that in general (1.1) does not admit a global interior Harnack inequality as it does for general linear models and for (1.1) in the case when $a(x)$ is a positive constant. Note that in the second case the positive solution can be decomposed into the form $\lambda \Theta_{\lambda}$ and that there exists a positive constant $c>0$ such that $\lim _{\lambda \uparrow \infty} \Theta_{\lambda}=c$ uniformly on compact subsets of $\Omega$ (cf. [8] for details). Figure 2.2 shows the global bifurcation diagram of positive solutions of (2.5), obtained by bringing together the three pieces on the first column of Figure 2.1, and a piece of the profiles of the positive solutions corresponding with the values $\lambda=124.9319,200.9235,230.3918,240.7325$ and 242.5004 to illustrate how the solutions stabilize to a limiting solution on the support of $a(x)$ and grow to infinity on its complement. The main goal of Sections 3 and 6 will be to show how these numerical predictions are true.

The following example shows that the monotonicity assumption imposed on the nonlinearity in the statement of Theorem 2.4 is necessary for its validity:

$$
\begin{gathered}
\Omega=(0,1), \quad r=4, \quad g(x, u)=11 u^{2}-6 u^{3}-6 u, \\
a(x)=\max \left\{0, \frac{4}{9}\left(-25 x^{2}+25 x-4\right) .\right.
\end{gathered}
$$

Using 63 modes we have computed the curve of positive solutions emanating from $u=0$ at $\lambda=\sigma_{1}^{\Omega}[-\Delta] \simeq 9.8696$ up to the value $\lambda=15$. For $\lambda>15$ the bifurcation diagram looks like the diagram of (2.5). Figure 2.3 shows the corresponding bifurcation diagram as well as the graphics of some representative solutions on it.

Now, the curve of positive solutions exhibits three turning points at the values $\lambda=9.1973,10.2233$ and 9.3071. The dimension of the unstable manifold of the positive solution changes by 1 each time that a turning point is passed. Stable positive solutions are represented by continuous lines, unstable positive solutions by dashed lines. The dimension of the unstable manifold of the unstable solutions is always 1 . As in (2.5), in the present example the positive solution grows to infinity in $\Omega_{0}$ while it stabilizes on $D$ to a limiting solution as $\lambda$ approaches the numerical $\sigma_{1}^{\Omega_{0}}[-\Delta]$. Notice that for each $\lambda \in(9.1973,9.8696)$ the problem possesses at least two positive solutions, one stable and the other unstable, and that for each $\lambda \in$ $(9.8696,10.2233)$, it possesses three positive solutions, two stable and one unstable. In fact, it possesses four solutions for each $\lambda \in(9.3071,9.8696)$, two stable and two unstable. In particular, condition (2.3) does not in general characterize the existence of a positive solution and, in addition, the uniqueness assertion of Theorem 2.4 might fail. What remains true for general nonlinearities is that under condition (2.3) the problem has a positive solution and that it does not admit a positive solution if $\lambda \geqq \sigma_{1}^{\Omega_{0}}[-\Delta]$. The following result makes precise these general features.

Theorem 2.5. Suppose that $a(x)$ and $g(x, u)$ satisfy $(\mathrm{H} a)$ and $(\mathrm{H} g \mathrm{w})$, respectively. Then (i) Problem (1.1) does not admit a positive solution if $\lambda \geqq \sigma_{1}^{\Omega_{0}}[-\Delta]$, and it possesses a positive solution if (2.3) is satisfied.

(ii) Let $\left\{u_{\lambda}\right\}$ be any family of positive solutions of $(1.1), \lambda \in\left(\sigma_{1}^{\Omega}[-\Delta], \sigma_{1}^{\Omega_{0}}[-\Delta]\right)$. Then,

$$
\lim _{\lambda \uparrow \sigma_{1}^{\Omega_{0}}[-\Delta]}\left\|u_{\lambda}\right\|_{L^{\infty}(\Omega)}=\infty .
$$



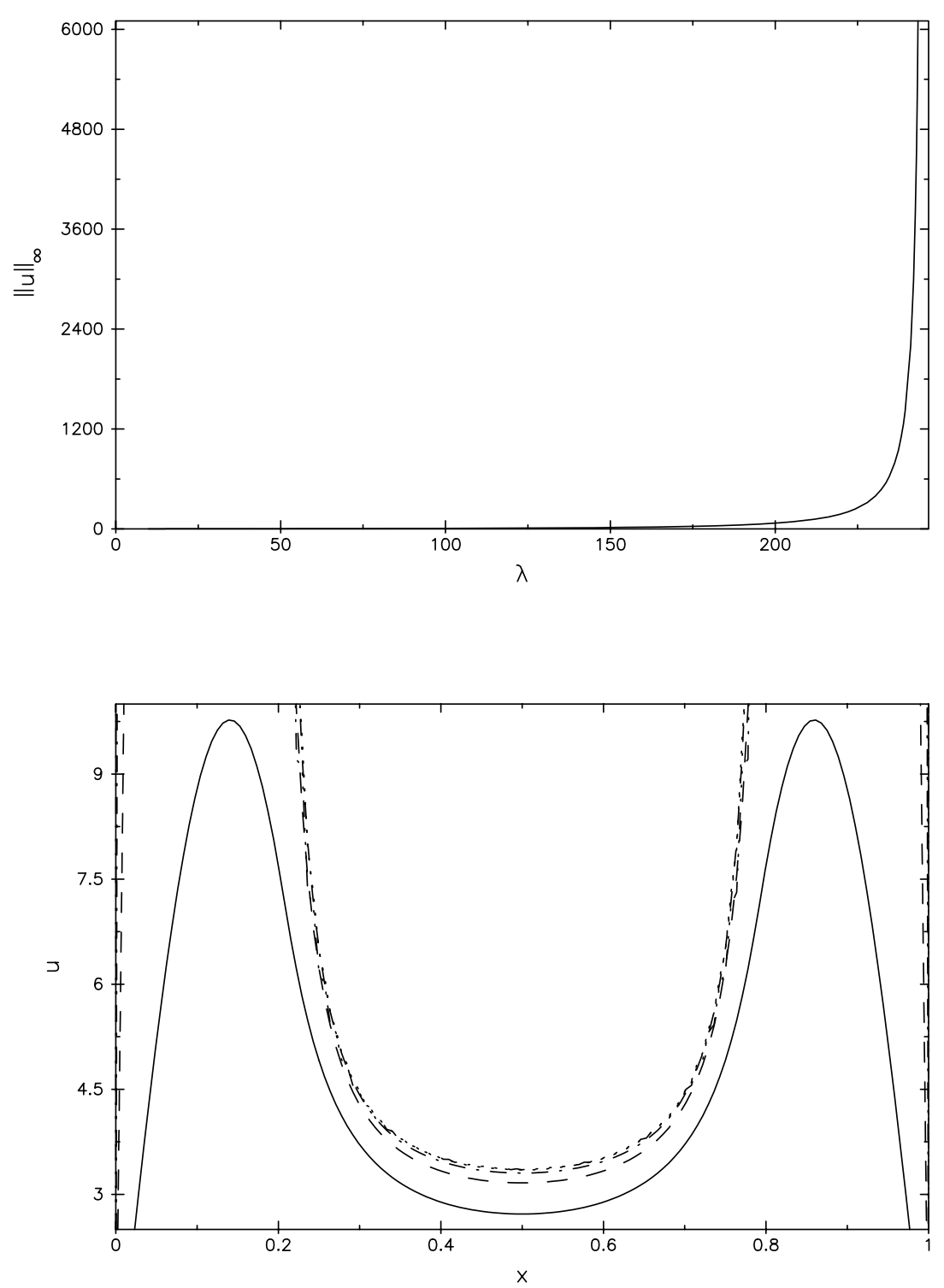

Fig. 2.2. Global bifurcation diagram and stabilization in $D$.

This result is new in all its generality. For smooth nonlinearities, (i) is contained in [9] and [13]. Here, we give a shorter different proof.

Proof. Let $u$ be a positive solution of (1.1). Then, $u$ is a positive eigenfunction of the operator $-\Delta+a(x)\left(u^{r}+g(x, u)\right)$ associated with the eigenvalue $\lambda$. Thus, by the uniqueness of the principal eigenvalue and its monotonicity with respect to the domain, we find that

$$
\lambda=\sigma_{1}^{\Omega}\left[-\Delta+a(x)\left(u^{r}+g(x, u)\right)\right]<\sigma_{1}^{\Omega_{0}}[-\Delta],
$$



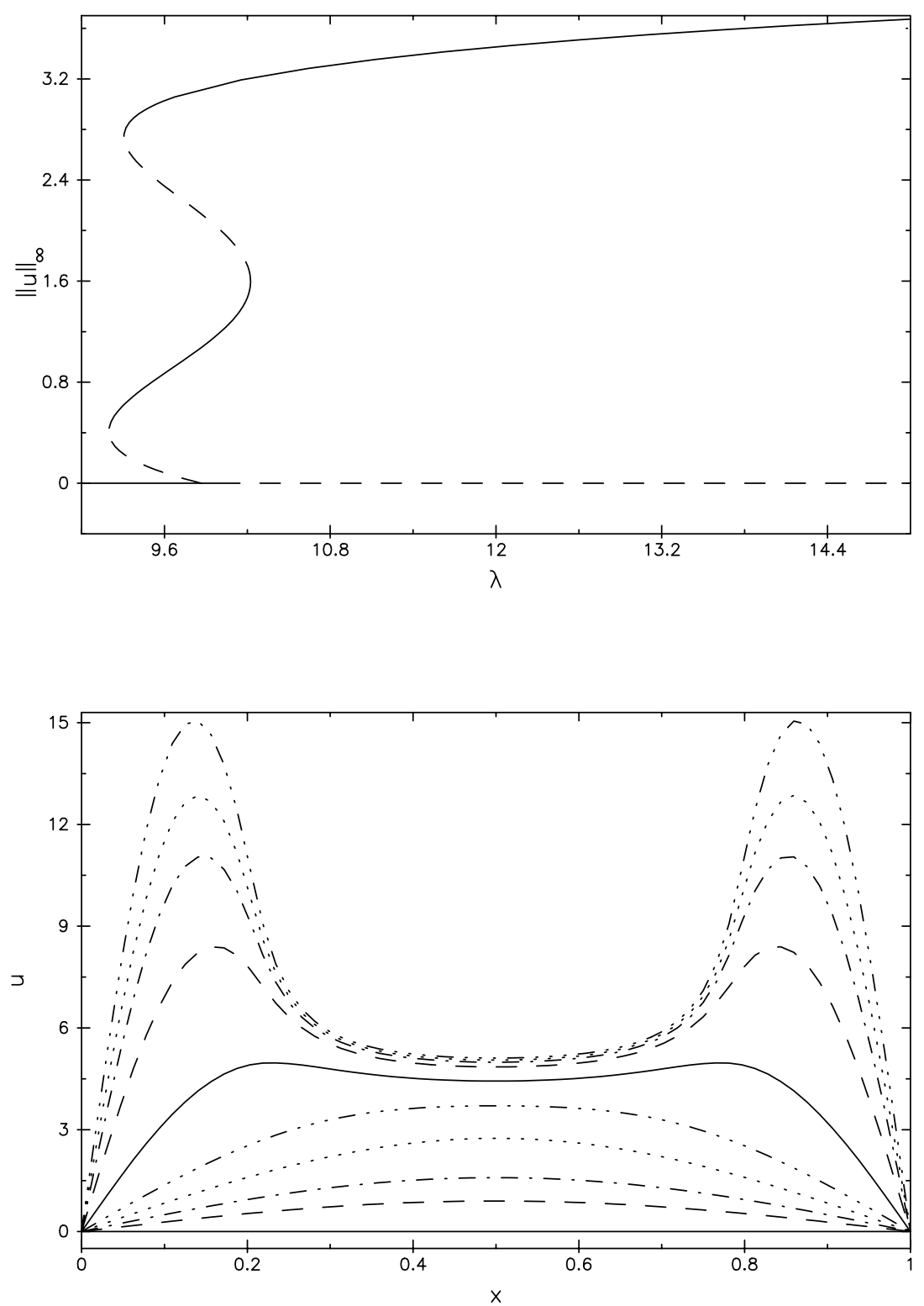

Fig. 2.3. Bifurcation diagram in the absence of monotonicity.

since $a=0$ on $\Omega_{0}$. Now, pick $\lambda$ satisfying (2.3). Let $\varphi$ denote the principal eigenfunction associated with $\sigma_{1}^{\Omega}[-\Delta]$. By $(\mathrm{H} g \mathrm{w})$, for $\varepsilon>0$ sufficiently small the function $\varepsilon \varphi$ provides us with a subsolution of (1.1) in the interior of the positive cone of $C_{0}^{1}(\bar{\Omega})$. Thus, to complete the proof of (i) it suffices to show the existence of a supersolution in the interior of this cone (cf. [1]). Fix $\varepsilon \in(0,1)$ and consider 
the auxiliary problem

$$
-\Delta u=\lambda u-(1-\varepsilon) a(x) u^{r+1} \quad \text { in } \Omega,\left.\quad u\right|_{\partial \Omega}=0 .
$$

Thanks to Theorem 2.4, $\theta_{[\lambda,(1-\varepsilon) a, 0]}$ is the unique positive solution of (2.8). Moreover, by $(\mathrm{Hgw})$ there is $u_{1}=u_{1}(\varepsilon)$ such that

$$
|g(x, u)| \leqq \varepsilon u^{r} \quad \forall(x, u) \in \bar{\Omega} \times\left[u_{1}, \infty\right) .
$$

Note that for any $c>1$ the function $\bar{u}:=c \theta_{[\lambda,(1-\varepsilon) a, 0]}$ is a supersolution of (2.8) in the interior of the positive cone. Let $c$ be so large that $\inf _{x \in D} \bar{u} \geqq u_{1}$. For this choice we have

$$
a \bar{u}\left(\bar{u}^{r}+g(x, \bar{u})\right) \geqq(1-\varepsilon) a \bar{u}^{r+1}
$$

for all $x \in \bar{\Omega}$ and hence

$$
-\Delta \bar{u} \geqq \lambda \bar{u}-(1-\varepsilon) a \bar{u}^{r+1} \geqq \lambda \bar{u}-a \bar{u}\left(\bar{u}^{r}+g(x, \bar{u})\right) .
$$

Therefore, $\bar{u}$ is a supersolution of (1.1) in the interior of the positive cone. This completes the proof of (i).

To show (ii) we argue by contradiction. If (2.7) fails, then there exists a constant $K$ and a sequence $\lambda_{n}, n \geqq 1$, such that $\lim _{n \rightarrow \infty} \lambda_{n}=\sigma_{1}^{\Omega_{0}}[-\Delta]$ and

$$
\left\|u_{\lambda_{n}}\right\|_{L^{\infty}(\Omega)} \leqq K
$$

for each $n \geqq 1$. Now, the same compactness argument as in the proof of Theorem 2.4 shows that (1.1) possesses a positive solution for $\lambda=\sigma_{1}^{\Omega_{0}}[-\Delta]$. By (i) this is impossible. This contradiction completes the proof.

\section{Pointwise Growth to Infinity on $\Omega_{0}$ and $\partial D$}

As already shown by Theorem 2.5, the $L^{\infty}$-norm of any solution of (1.1) grows to infinity as $\lambda \uparrow \sigma_{1}^{\Omega_{0}}[-\Delta]$ and it ceases to exist for $\lambda \geqq \sigma_{1}^{\Omega_{0}}[-\Delta]$. In this section we explain this explosive character by analyzing the pointwise growth of solutions. As an immediate consequence, our analysis entails that any solution of (1.1) must grow to infinity uniformly on compact subsets of $\Omega_{0} \cup \partial D$ as $\lambda \uparrow \sigma_{1}^{\Omega_{0}}[-\Delta]$. In Section 6 the optimality of this result is shown to arise from the fact that any positive solution must approach a limiting solution in $D$. These results agree with the numerical calculations already done in Section 2. Some previous results in this direction were given in [15] for the very special case when $g=0$. Our first result provides us with the pointwise divergence in $\Omega_{0}$.

Theorem 3.1. Suppose that $a(x)$ and $g(x, u)$ satisfy $(\mathrm{H} a)$ and $(\mathrm{H} g \mathrm{w})$, respectively. Let $\left\{u_{\lambda}\right\}$ denote any family of positive solutions of $(1.1), \lambda \in\left(\sigma_{1}^{\Omega}[-\Delta], \sigma_{1}^{\Omega_{0}}[-\Delta]\right)$. Then,

$$
\lim _{\lambda \uparrow \sigma_{1}^{\Omega_{0}}[-\Delta]} u_{\lambda}(x)=\infty \quad \forall x \in \Omega_{0} .
$$


In the proof of this theorem we shall use a technical lemma which is of interest in its own right:

Lemma 3.2. Let $\left\{u_{\lambda}\right\}$ be a family of positive solutions of (1.1), $\sigma_{1}^{\Omega}[-\Delta]<\lambda<$ $\sigma_{1}^{\Omega_{0}}[-\Delta]$, and pick $\lambda_{1} \in\left(\sigma_{1}^{\Omega}[-\Delta], \sigma_{1}^{\Omega_{0}}[-\Delta]\right)$. Then, there exists a positive constant $c>0$ such that

$$
\inf _{x \in D} u_{\lambda}(x) \geqq c>0 \quad \forall \lambda \geqq \lambda_{1} .
$$

Proof. We first show that there exists $\hat{g} \in C(\bar{\Omega} \times[0, \infty))$ locally Lipschitz continuous with respect to $u$ such that $g \leqq \hat{g}, \hat{g}(\cdot, 0)=0, u \rightarrow u^{r}+\hat{g}(\cdot, u)$ is increasing and

$$
\lim _{u \uparrow \infty}\left(u^{r}+\hat{g}(\cdot, u)\right)=\infty \quad \text { uniformly in } \bar{\Omega} .
$$

By (2.2), there exists $u_{0}>0$ and $L>0$ such that $g(x, u) \leqq \frac{1}{2} u^{r}$ for $u \geqq u_{0}$ and $x \in \bar{\Omega}$, and $g(x, u) \leqq L u$ for $0 \leqq u<u_{0}$ and $x \in \bar{\Omega}$. Taking any continuous increasing function $\hat{g}(x, u)=\hat{g}(u)$ such that $\hat{g}(0)=0, \hat{g}(u) \geqq L u$ for $u \in\left[0, u_{0}\right]$ and $\hat{g}(u) \geqq \frac{1}{2} u^{r}$ for $u \geqq u_{0}$ we are done.

Since $\hat{g} \geqq g$, any positive solution of (1.1) is a positive supersolution of

$$
-\Delta v=\lambda v-a(x)\left(v^{r}+\hat{g}(x, v)\right) v \quad \text { in } \Omega,\left.\quad v\right|_{\partial \Omega}=0,
$$

which is a problem satisfying all the requirements of Theorem 2.4. By the uniqueness of the positive solution, the strong maximum principle gives $u_{\lambda}>\theta_{[\lambda, a, \hat{g}]}$. Moreover, thanks to Theorem 2.4(iii), $\theta_{[\lambda, a, \hat{g}]} \geqq \theta_{\left[\lambda_{1}, a, \hat{g}\right]}$ provided that

$$
\lambda \in\left[\lambda_{1}, \sigma_{1}^{\Omega_{0}}[-\Delta]\right)
$$

Since $\bar{D} \subset \Omega$ and $\theta_{\left[\lambda_{1}, a, \hat{g}\right]}$ lies in the interior of the positive cone of $C_{0}^{1}(\bar{\Omega})$, making the choice $c:=\inf _{\bar{D}} \theta_{\left[\lambda_{1}, a, \hat{g}\right]}$ completes the proof.

Proof of Theorem 3.1. Pick $\lambda_{1} \in\left(\sigma_{1}^{\Omega}[-\Delta], \sigma_{1}^{\Omega_{0}}[-\Delta]\right)$ and let $c>0$ be the constant given by Lemma 3.2. By (2.2) there is $M>0$ such that

$$
g(x, u) \leqq M u^{r} \quad \text { for each } x \in \Omega \text { and } u \geqq c .
$$

Then, since $a=0$ in $\Omega \backslash D$, for each $\lambda \geqq \lambda_{1}$ and $x \in \bar{\Omega}$ we have that

$$
a(x)\left(u_{\lambda}^{r}(x)+g\left(x, u_{\lambda}(x)\right)\right) \leqq a(x)(M+1) u_{\lambda}^{r}(x),
$$

and hence, $u_{\lambda}$ is a positive supersolution of

$$
-\Delta v=\lambda v-(M+1) a(x) v^{r+1} \quad \text { in } \Omega,\left.\quad v\right|_{\partial \Omega}=0,
$$

for each $\lambda \geqq \lambda_{1}$. Thus,

$$
u_{\lambda} \geqq \theta_{[\lambda, a(M+1), 0]}=(M+1)^{-1 / r} \theta_{[\lambda, a, 0]},
$$


and it suffices to show that

$$
\lim _{\lambda \uparrow \sigma_{1}^{\Omega_{0}}[-\Delta]} \theta_{[\lambda, a, 0]}(x)=\infty
$$

for each $x \in \Omega_{0}$. Although this relation was shown to occur in [15], for the sake of completeness we outline its proof here. Differentiating the $\theta_{[\lambda, a, 0]}$-equation with respect to $\lambda$ gives

$$
\left(-\Delta-\lambda+(r+1) a(x) \theta_{[\lambda, a, 0]}^{r}\right) \frac{d \theta_{[\lambda, a, 0]}}{d \lambda}=\theta_{[\lambda, a, 0]} \quad \text { in } \Omega,\left.\quad \theta_{[\lambda, a, 0]}\right|_{\partial \Omega}=0 .
$$

Moreover, for $\lambda \geqq \lambda_{1}$ we have $\theta_{[\lambda, a, 0]} \geqq \theta_{\left[\lambda_{1}, a, 0\right]}$ and hence there exists a constant $c_{1}>0$ such that

$$
\theta_{[\lambda, a, 0]} \geqq c_{1} \psi \quad \text { in } \Omega,
$$

where $\psi(x)$ is the principal eigenfunction associated with $\sigma_{1}^{\Omega_{0}}[-\Delta]$. Thus, the strong maximum principle implies that $\frac{d \theta_{[\lambda, a, 0]}}{d \lambda}>w_{\lambda}$ for each $\lambda>\lambda_{1}$, where $w_{\lambda}$ is the unique positive solution of

$$
(-\Delta-\lambda) w_{\lambda}=c_{1} \psi \quad \text { in } \Omega_{0},\left.\quad w_{\lambda}\right|_{\partial \Omega_{0}}=0,
$$

which is given by $w_{\lambda}=\left(\sigma_{1}^{\Omega_{0}}[-\Delta]-\lambda\right)^{-1} \psi_{1}$. Therefore,

$$
\lim _{\lambda \uparrow \sigma_{1}^{\Omega_{0}}[-\Delta]} \frac{d \theta_{[\lambda, a, 0]}}{d \lambda}(x)=\infty, \quad \forall x \in \Omega_{0} .
$$

This shows (3.5) and completes the proof.

In Section 6 we shall show how the family $\left\{u_{\lambda}\right\}$ is uniformly bounded above on any compact subset of $D$ as $\lambda \uparrow \sigma_{1}^{\Omega_{0}}[-\Delta]$. So, in order to acquire a complete understanding of the pointwise growth of the positive solutions of (1.1) at $\sigma_{1}^{\Omega_{0}}[-\Delta]$, it only remains to analyze the limiting behavior of the family at the interphase $\partial D$. Actually, for the very special case when $g=0$ this analysis was carried out in [15], where some sufficient conditions on the decay to zero of $a(x)$ were given so that (3.1) holds for each $x \in \partial D$. Based on these results, the argument of the proof of Theorem 3.1 provides us with the following result.

Corollary 3.3. Assume that $a(x)$ and $g(x, u)$ satisfy $(\mathrm{H} a)$ and $(\mathrm{H} g \mathrm{w})$, respectively, and that in addition each component of $D$ is a $C^{3}$ subdomain of $\Omega$ and

$$
\lim _{\operatorname{dist}(x, \partial D) \downarrow 0} \frac{a(x)}{\operatorname{dist}(x, \partial D)}=0 .
$$

Then,

$$
\lim _{\lambda \uparrow \sigma_{1}^{\Omega_{0}}[-\Delta]} u_{\lambda}(x)=\infty \quad \text { uniformly on } \partial D .
$$

Proof. Theorem 4.3 of [15] implies that condition (3.7) is satisfied if $g=0$. This corollary is then an immediate consequence of estimate (3.4).

Remark 3.4. If $a(x)$ is assumed to be of class $C^{1}$ near $\partial D$, then condition (3.6) is satisfied. 


\section{On the Uniqueness of the Positive Solution}

In this section we give some sufficient conditions for the uniqueness of the positive solution of (1.1) in the general case when $g(x, u)$ satisfies $(\mathrm{H} g)$. As has been noted in the Introduction, the positive solutions of (1.1) for $\lambda$ close to $\sigma_{1}^{\Omega}[-\Delta]$ bifurcate from the state $u=0$. Moreover, by using singularity theory, it is easy to give examples exhibiting any predetermined number of positive solutions in a neighborhood of the bifurcation point. Figure 2.3 shows one of the possible bifurcation diagrams. Our uniqueness results show how the complexity of these bifurcation diagrams diminishes as $\lambda$ is taken progressively closer to $\sigma_{1}^{\Omega_{0}}[-\Delta]$, the value where bifurcation from infinity occurs. Indeed, Figure 2.3 suggests that the model (2.6) possesses a unique positive solution for $\lambda$ sufficiently close to $\sigma_{1}^{\Omega_{0}}[-\Delta]$. Our main uniqueness result establishes that this is in fact the case for the general problem (1.1) provided that the support $D$ of the weight function $a(x)$ satisfies a geometrical condition involving its thickness. To state the main theorem we have first to introduce that concept. Given $z \in \mathbb{R}^{N}, l>0$, and $v \in \mathbb{R}^{N}$ with $|v|=1$, consider the slice $S_{z, v, l}$ based upon $z$, with thickness $l$ in the direction of $v$ :

$$
S_{z, v, l}:=\left\{x \in \mathbb{R}^{N}: 0<(x-z) \cdot v<l\right\} .
$$

Then, the thickness $T:=T(D)$ of $D$ is defined by

$$
T(D):=\inf \left\{l>0: D \subset S_{z, v, l} \text { for some } z, v \in \mathbb{R}^{N},|v|=1\right\} .
$$

In other words, $T(D)$ is the minimum distance between parallel hyperplanes enclosing $D$. We are now ready to state our first uniqueness result.

Theorem 4.1. Assume that $a(x)$ and $g(x, u)$ satisfy $(\mathrm{H} a)$ and $(\mathrm{H} g)$, respectively, and let $T$ be the thickness of $D$. Then, there exists $\varepsilon_{0}>0$ such that if

$$
T^{2}\|a\|_{L^{\infty}(\Omega)} \leqq \varepsilon_{0},
$$

then there exists $\delta>0$ with the property that (1.1) possesses a unique positive solution for each $\lambda \in\left[\sigma_{1}^{\Omega_{0}}[-\Delta]-\delta, \sigma_{1}^{\Omega_{0}}[-\Delta]\right)$.

Remark 4.2. Condition (4.1) can be reached either by fixing the amplitude of $a(x)$ and taking $T(D)$ sufficiently small, or by fixing $T(D)$ and taking $a(x)$ with sufficiently small amplitude. Therefore, this result provides us with a substantial improvement of the main theorem of [13], where the uniqueness was shown to occur for $a(x)$ sufficiently small.

Proof of Theorem 4.1. Pick $\lambda_{0} \in\left(\sigma_{1}^{\Omega}[-\Delta], \sigma_{1}^{\Omega_{0}}[-\Delta]\right)$. Thanks to Lemma 3.2 and the first part of the proof of Theorem 3.1, there exists a constant $M=M\left(\lambda_{0}\right)>0$ such that

$$
u \geqq M^{-1 / r} \theta_{[\lambda, a, 0]}
$$

for any positive solution $u$ of (1.1) with $\lambda \geqq \lambda_{0}$. Now, fix $\varepsilon>0$ sufficiently small and consider the open set

$$
D^{\varepsilon}:=\{x \in \Omega: \operatorname{dist}(x, D)<\varepsilon\} .
$$


Since $\partial D^{\varepsilon} \subset \Omega_{0}$, Theorem 3.1 implies that

$$
\lim _{\lambda \uparrow \sigma_{1}^{\Omega_{0}}[-\Delta]} \theta_{[\lambda, a, 0]}(x)=\infty \quad \text { uniformly on } \partial D^{\varepsilon} .
$$

Moreover, since $\lambda \rightarrow \theta_{[\lambda, a, 0]}$ is pointwise increasing, for each $n \in \mathbb{N}$ there exists $\lambda_{n} \in\left(\lambda_{0}, \sigma_{1}^{\Omega_{0}}[-\Delta]\right), \lambda_{n}<\lambda_{n+1}$, such that

$$
\theta_{[\lambda, a, 0]}>n \quad \text { on } \partial D^{\varepsilon} \quad \forall \lambda \in\left[\lambda_{n}, \sigma_{1}^{\Omega_{0}}[-\Delta]\right) \text {. }
$$

Necessarily $\lim _{n \rightarrow \infty} \lambda_{n}=\sigma_{1}^{\Omega_{0}}[-\Delta]$, and it follows from (4.4) that if $\lambda \geqq \lambda_{n}$, then $\theta_{[\lambda, a, 0]}$ is a positive supersolution of the problem

$$
-\Delta v=\lambda v-\|a\|_{L^{\infty}(\Omega)} v^{r+1} \quad \text { in } D^{\varepsilon},\left.\quad v\right|_{\partial D^{\varepsilon}}=n .
$$

We now show that (4.5) has a unique positive solution for each $\lambda \in\left[\lambda_{0}, \sigma_{1}^{\Omega_{0}}[-\Delta]\right.$ ) provided that $n \geqq\left(\lambda_{0} /\|a\|_{L^{\infty}(\Omega)}\right)^{1 / r}$. To prove the uniqueness, assume that $v_{1}, v_{2}$ are two positive solutions of (4.5). Then,

$$
\left(-\Delta-\lambda+\|a\|_{L^{\infty}(\Omega)} \frac{v_{1}^{r+1}-v_{2}^{r+1}}{v_{1}-v_{2}}\right)\left(v_{1}-v_{2}\right)=0 \quad \text { in } D^{\varepsilon},\left.\left(v_{1}-v_{2}\right)\right|_{\partial D^{\varepsilon}}=0,
$$

and moreover there is $R \geqq 0, R \neq 0$, such that

$$
\frac{v_{1}^{r+1}-v_{2}^{r+1}}{v_{1}-v_{2}}=v_{1}^{r}+R(x) \text {. }
$$

Thus, by the monotonicity of the principal eigenvalue with respect to the potential, we find that

$$
\sigma_{1}^{D^{\varepsilon}}\left[-\Delta-\lambda+\|a\|_{L^{\infty}(\Omega)} \frac{v_{1}^{r+1}-v_{2}^{r+1}}{v_{1}-v_{2}}\right]>\sigma_{1}^{D^{\varepsilon}}\left[-\Delta-\lambda+\|a\|_{L^{\infty}(\Omega)} v_{1}^{r}\right] .
$$

On the other hand, since $v_{1}$ is a solution of (4.5), it provides us with a strict positive supersolution of the operator $-\Delta-\lambda+\|a\|_{L^{\infty}(\Omega)} v_{1}^{r}$ in $D^{\varepsilon}$ subject to homogeneous Dirichlet boundary conditions and hence, the strong maximum principle implies that $\sigma_{1}^{D^{\varepsilon}}\left[-\Delta-\lambda+\|a\|_{L^{\infty}(\Omega)} v_{1}^{r}\right]>0$. Thus, due to (4.7),

$$
\sigma_{1}^{D^{\varepsilon}}\left[-\Delta-\lambda+\|a\|_{L^{\infty}(\Omega)} \frac{v_{1}^{r+1}-v_{2}^{r+1}}{v_{1}-v_{2}}\right]>0
$$

and we find from (4.6) that $v_{1}=v_{2}$, which is impossible. This shows the uniqueness of the positive solution of (4.5), if it exists. Note that in this argument $v_{1}$ does not need to be the minimal positive solution. To prove the existence of a solution we use the method of sub- and supersolutions. Sufficiently large constants provide us with positive supersolutions. It remains to show the existence of a positive subsolution. To construct it we use functions of traveling-wave type:

$$
v(x)=w(\zeta), \quad \zeta=\left(x_{0}-x\right) \cdot n\left(x_{0}\right),
$$


where $x_{0} \in \partial D^{\varepsilon}$ and $n\left(x_{0}\right)$ stands for the outward unit normal to $D^{\varepsilon}$ at $x_{0}$. Since $T\left(D^{\varepsilon}\right)=T(D)+2 \varepsilon$, there exists $x_{0} \in \partial D^{\varepsilon}$ such that $D^{\varepsilon} \subset S_{x_{0},-n\left(x_{0}\right), T(D)+2 \varepsilon}$. Moreover, the function $v(x)$ defined by (4.8) satisfies the $v$-equation of (4.5) in $\bar{D}^{\varepsilon}$ if and only if $w(\zeta)$ satisfies

$$
-\frac{d^{2} w}{d \zeta^{2}}=\lambda w-\|a\|_{L^{\infty}(\Omega)} w^{r+1}, \quad 0 \leqq \zeta \leqq T(D)+2 \varepsilon .
$$

To complete the proof of the existence it suffices to show that (4.9) admits a solution $w(\zeta)$ with

$$
w(0)=w(T(D)+2 \varepsilon)=n .
$$

This is so because any solution of (4.9), (4.10) satisfies $w(\zeta)<n$ for each $\zeta \in$ $(0, T(D)+2 \varepsilon)$ and hence, the associated $v(x)$ satisfies $v \leqq n$ on $\partial D^{\varepsilon}$. The existence of a positive solution to (4.9), (4.10) is guaranteed by the following lemma whose proof is postponed until the end of the proof of Theorem 4.1.

Lemma 4.3. Consider the one-dimensional problem

$$
-u^{\prime \prime}=\lambda u-A u^{r+1} \quad \text { in }(0, T), \quad u(0)=u(T)=\sigma,
$$

where $\lambda>0, A>0, r>0, T>0$ and $\sigma \in\left[(\lambda / A)^{1 / r}, \infty\right]$. Then, (4.11) possesses a unique solution, denoted by $U_{\sigma}(\zeta)$, which is positive and pointwise increasing with respect to $\sigma$. Moreover, the solution $U_{\infty}$ corresponding to $\sigma=\infty$ is finite in $0<\zeta<L$ and is given by $U_{\infty}(\zeta)=\lim _{\sigma \uparrow \infty} U_{\sigma}(\zeta)$. Furthermore,

$$
\frac{2 B_{0}^{2}}{(r+2) A T^{2}} \leqq \min _{\zeta \in(0, L)} U_{\infty}^{r}(\zeta) \leqq \frac{2 B_{0}^{2}}{(r+2) A T^{2}}+\frac{\lambda(r+2)}{2 A},
$$

where $B_{0}=B\left(\frac{r}{2(r+2)}, \frac{1}{2}\right)$ and $B(p, q)=\int_{0}^{1} t^{p-1}(1-t)^{q-1} d t$ is Euler's function.

Thanks to Lemma 4.3, the problem (4.9), (4.10) possesses a unique solution $w_{n}:=U_{n}(\zeta)$, necessarily positive, provided that $n \geqq\left(\lambda /\|a\|_{L^{\infty}(\Omega)}\right)^{1 / r}$. Moreover, thanks to (4.12), the corresponding subsolution of (4.5), $v_{n}(x)$ given from $w_{n}$ by (4.8), satisfies

$$
\inf _{D^{\varepsilon}} v_{n} \geqq\left(\frac{2 B_{0}^{2}}{\|a\|_{L^{\infty}(\Omega)}(r+2)(T+2 \varepsilon)^{2}}\right)^{1 / r} .
$$

Now, by the uniqueness of the positive solution of (4.5), there exists $n_{0} \in \mathbb{N}$ such that for each $\lambda \in\left[\lambda_{n_{0}}, \sigma_{1}^{\Omega_{0}}[-\Delta]\right)$ we have $\theta_{[\lambda, a, 0]} \geqq v_{n_{0}}$ and therefore, thanks to (4.2) and passing to the limit as $\varepsilon \downarrow 0$, we find from (4.13) that

$$
\inf _{D} u \geqq\left(\frac{2 B_{0}^{2}}{(r+2) M T^{2}\|a\|_{L^{\infty}(\Omega)}}\right)^{1 / r}
$$

for any positive solution $u(x)$ of (1.1) with $\lambda \geqq \lambda_{n_{0}}$. Thanks to (4.14), $\inf _{D} u$ is as large as we want if $T^{2}\|a\|_{L^{\infty}(\Omega)}$ is sufficiently small. By ( $\left.\mathrm{Hg}\right)$ there exists $u_{0}>0$ such that $u \rightarrow u^{r}+g(x, u)$ is increasing in $\left[u_{0}, \infty\right)$ for each $x \in \bar{\Omega}$. By (4.14) there 
exists $\varepsilon_{0}>0$ such that (4.1) implies that $\inf _{D} u \geqq u_{0}$ for any positive solution of (1.1) with $\lambda \geqq \lambda_{n_{0}}$. It remains to show that $\varepsilon_{0}$ satisfies the statement of the theorem. To prove this we use a device from [3]. It was shown in [3] that for any pair $u_{1}, u_{2}$ of solutions of (1.1) with $u_{1} / u_{2}, u_{2} / u_{1} \in L^{\infty}(\Omega)$ the following inequality holds

$$
I\left(u_{1}, u_{2}\right):=\int_{\Omega}\left(-\frac{\Delta u_{1}}{u_{1}}+\frac{\Delta u_{2}}{u_{2}}\right)\left(u_{1}^{2}-u_{2}^{2}\right) d x \geqq 0 .
$$

By the Hopf Lemma, this inequality is true for any pair of positive solutions. Thus, if $u_{1}, u_{2}$ are two positive solutions of (1.1), then

$$
I\left(u_{1}, u_{2}\right)=\int_{D} a(x)\left(g\left(x, u_{2}\right)-g\left(x, u_{1}\right)+u_{2}^{r}-u_{1}^{r}\right)\left(u_{1}^{2}-u_{2}^{2}\right) d x \geqq 0,
$$

since $a=0$ in $\Omega \backslash D$. Now, pick $\lambda \geqq \lambda_{n_{0}}$ and assume that (1.1) possesses two positive solutions, say, $u_{1}$ and $u_{2}$. By the construction of $\varepsilon_{0}$, condition (4.1) implies

$$
a(x)\left(g\left(x, u_{1}\right)-g\left(x, u_{2}\right)+u_{1}^{r}-u_{2}^{r}\right)\left(u_{1}^{2}-u_{2}^{2}\right) \geqq 0 \quad \forall x \in \Omega .
$$

Thus, $I\left(u_{1}, u_{2}\right) \leqq 0$, and thanks to $(4.15), I\left(u_{1}, u_{2}\right)=0$. Hence, there exists $\gamma>0$ such that $u_{2}=\gamma u_{1}$ in $\Omega$ (cf. [3] for details). Therefore,

$$
g\left(x, \gamma u_{1}(x)\right)+\gamma^{r} u_{1}^{r}(x)=g\left(x, u_{1}(x)\right)+u_{1}^{r}(x) \text { for each } x \in D,
$$

which is impossible unless $\gamma=1$. This contradicts $u_{1} \neq u_{2}$ and completes the proof of the theorem.

Proof of Lemma 4.3. We look at the phase portrait of the planar first-order system associated with the differential equation of (4.11). The ' stands for differentiation with respect to $\zeta$. With $v=u^{\prime}$, the orbits of the system are described by the family of curves

$$
\frac{1}{2} v^{2}+\frac{\lambda}{2} u^{2}-\frac{A}{r+2} u^{r+2}=E, \quad E \in \mathbb{R}
$$

and two regimes are possible: Either $0<\sigma<(\lambda / A)^{1 / r}$ or $\sigma \geqq(\lambda / A)^{1 / r}$. By the assumptions of the lemma we are restricting ourselves to deal with the second one. If $\sigma=(\lambda / A)^{1 / r}$, then $u=(\lambda / A)^{1 / r}$ provides us with the unique solution of (4.11), since this value corresponds to an equilibrium. Hence, in the sequel it is assumed that $\sigma>(\lambda / A)^{1 / r}$. In this case, by simply looking at the phase portrait, it is easily seen that $u_{0} \in \mathbb{R}$ corresponds to the minimum of a solution of (4.11) if and only if

$$
(\lambda / A)^{1 / r}<u_{0}<\sigma, \quad \frac{1}{2} T=\int_{u_{0}}^{\sigma}\left(\frac{2 A u^{r+2}}{r+2}-\lambda u^{2}+\lambda u_{0}^{2}-\frac{2 A u_{0}^{r+2}}{r+2}\right)^{-1 / 2} d u
$$

i.e., if and only if

$$
\left(\frac{\lambda}{A}\right)^{1 / r}<u_{0}<\sigma, \quad \frac{1}{2} T=J\left(\sigma, u_{0}\right)
$$


where

$$
J\left(\sigma, u_{0}\right):=\int_{1}^{\sigma / u_{0}}\left[\lambda\left(1-t^{2}\right)+\frac{2 A}{r+2} u_{0}^{r}\left(t^{r+2}-1\right)\right]^{-1 / 2} d t .
$$

Thus, the solutions of (4.11) are in one-to-one correspondence with the solutions of (4.16). Since $J\left(\sigma, u_{0}\right)$ is decreasing in $u_{0}$ and

$$
\lim _{u_{0} \downarrow(\lambda / A)^{1 / r}} J\left(\sigma, u_{0}\right)=\infty, \quad \lim _{u_{0} \uparrow \sigma} J\left(\sigma, u_{0}\right)=0,
$$

there exists a unique value of $u_{0}$, say, $u_{0}(\sigma)$, satisfying (4.16). Therefore, (4.11) possesses a unique solution, denoted in the sequel by $u=U_{\sigma}(\zeta)$.

We now construct the solution corresponding to $\sigma=\infty$. From (4.16), it is easily seen that $u_{0}(\sigma)=\min _{(0, T)} U_{\sigma}$ is increasing in $\sigma$. In fact, the strong maximum principle implies that $U_{\sigma}$ is pointwise increasing in $\sigma$. Now, assume that $\lim _{\sigma \uparrow \infty} u_{0}(\sigma)=\infty$. Then, we find from (4.16) and (4.17) that

$$
0<\frac{T}{2}=J\left(\sigma, u_{0}(\sigma)\right)<\int_{1}^{\infty}\left[\lambda\left(1-t^{2}\right)+\frac{2 A}{r+2} u_{0}^{r}(\sigma)\left(t^{r+2}-1\right)\right]^{-1 / 2} d t,
$$

while a simple calculation yields

$$
\int_{1}^{\infty}\left[\lambda\left(1-t^{2}\right)+\frac{2 A}{r+2} u_{0}^{r}\left(t^{r+2}-1\right)\right]^{-1 / 2} d t \sim\left(\frac{r+2}{2 A}\right)^{1 / 2} B_{1} u_{0}^{-r / 2} \downarrow 0,
$$

as $u_{0} \uparrow \infty$, where $B_{1}=\int_{1}^{\infty}\left(t^{r+2}-1\right)^{-1 / 2} d t$. This contradiction shows that $\lim _{\sigma \uparrow \infty} u_{0}(\sigma)$ is finite. Similarly, $\lim _{\sigma \uparrow \infty} U_{\sigma}(\zeta)$ is finite for each $\zeta \in(0, T)$. From these features, it is easily seen that the pointwise limit

$$
U_{\infty}(\zeta):=\lim _{\sigma \uparrow \infty} U_{\sigma}(\zeta), \quad \zeta \in(0, T),
$$

provides us with the unique solution of (4.11) for $\sigma=\infty$, which is characterized by $u_{0}(\infty):=\lim _{\sigma \uparrow \infty} u_{0}(\sigma)$, which is the unique solution of

$$
\frac{1}{2} T=J\left(\infty, u_{0}(\infty)\right) .
$$

Finally, the estimates in (4.12) follow readily from

$$
\begin{aligned}
\left(\frac{2 A u_{0}^{r}(\infty)}{r+2}-\lambda\right)\left(t^{r+2}-1\right) & \leqq \lambda\left(1-t^{2}\right)+\frac{2 A u_{0}^{r}(\infty)}{r+2}\left(t^{r+2}-1\right) \\
& \leqq \frac{2 A u_{0}^{r}(\infty)}{r+2}\left(t^{r+2}-1\right),
\end{aligned}
$$

which is valid for all $t \geqq 1$. This completes the proof.

We complete this section by giving another uniqueness result valid for a special class of $g(x, u)$ 's. Beside its own interest, since no further restrictions on the weight amplitude nor on the thickness of $D$ are needed for it, it will be one of the most important tools of Section 6 to analyze the behavior in $D$ of the positive solutions of (1.1) as $\lambda \uparrow \sigma_{1}^{\Omega_{0}}[-\Delta]$. 
Theorem 4.4. Assume that $a(x)$ satisfies $(\mathrm{H} a)$ and pick $\delta \in(0, r)$ and $b \in \mathbb{R}$. Then, there exists $\lambda_{0} \in\left(\sigma_{1}^{\Omega}[-\Delta], \sigma_{1}^{\Omega_{0}}[-\Delta]\right)$ such that the problem

$$
-\Delta u=\lambda u-a(x) u\left(u^{r}-b u^{r-\delta}\right) \quad \text { in } \Omega,\left.\quad u\right|_{\partial \Omega}=0,
$$

possesses a unique positive solution for each $\lambda_{0} \leqq \lambda<\sigma_{1}^{\Omega_{0}}[-\Delta]$.

Proof. Thanks to Theorem 2.4, we can assume $b>0$. In this case, for fixed $\varepsilon>0$ sufficiently small, it follows from Theorem 3.1 that there exists $\lambda_{0} \in$ $\left(\sigma_{1}^{\Omega}[-\Delta], \sigma_{1}^{\Omega_{0}}[-\Delta]\right)$ such that $u>b^{1 / \delta}$ on $\partial D^{\varepsilon}$ for any positive solution of (4.20) with $\lambda \geqq \lambda_{0}$, where $D^{\varepsilon}:=\{x \in \Omega$ : dist $(x, D)<\varepsilon\}$. Moreover, multiplying (4.20) by $\left(b^{1 / \delta}-u\right)^{+} \in H_{0}^{1}\left(D^{\varepsilon}\right)$ and integrating on $D^{\varepsilon}$ leads to

$$
\begin{aligned}
& -\int_{D_{\varepsilon}}\left|\nabla\left(b^{1 / \delta}-u\right)^{+}\right|^{2} d x \\
& =\int_{\left\{u \leqq b^{1 / \delta}\right\} \cap D^{\varepsilon}} u\left(b^{1 / \delta}-u\right)\left(\lambda-a(x) u^{r-\delta}\left(u^{\delta}-b\right)\right) d x \geqq 0,
\end{aligned}
$$

and hence, $\left(b^{1 / \delta}-u\right)^{+}=0$ a.e. in $D^{\varepsilon}$. Therefore, since $u$ is continuous, we find that

$$
u \geqq b^{1 / \delta} \quad \text { on } \bar{D},
$$

for any positive solution of (4.20) with $\lambda \geqq \lambda_{0}$. Finally, since the mapping $u \rightarrow$ $u^{r}-b u^{r-\delta}$ is increasing in $u \geqq b^{1 / \delta}$, the device of [3] used in the last part of the proof of Theorem 4.1 shows that (4.20) possesses a unique solution for each $\lambda \geqq \lambda_{0}$.

\section{On the Optimality of the Main Uniqueness Result}

In this section we use an example to show how Theorem 4.1 is optimal in the sense that there are problems such that for fixed $T$, an increase in $\|a\|_{L^{\infty}}(\Omega)$ produces a decrease in the range of $\lambda$ 's for which (1.1) has unique solutions. To make this analysis we consider a variant of problem (2.6),

$$
\begin{gathered}
\Omega=(0,1), \quad r=4, \quad g(x, u)=11 u^{2}-6 u^{3}-6 u, \\
a(x)=\varepsilon \max \left\{0, \frac{4}{9}\left(-25 x^{2}+25 x-4\right),\right.
\end{gathered}
$$

where $\varepsilon>0$ is regarded as a real parameter, and we compute the curve of positive solutions for the values $\varepsilon=1,10,50$ and 100. Figure 5.1 shows the bifurcation diagrams for each of these values of $\varepsilon$. We have represented the solutions for values of $\lambda<45$. The numerical calculations have been carried out as already explained in Section 2.

The folds of the bifurcation diagrams are magnified as the weight amplitude is increased. The case $\varepsilon=1$ corresponds to the example (2.6) of Section 2. In this case the numerics suggest that (5.1) possesses a unique positive solution for each $\lambda>10.2233$, which is the value of $\lambda$ where the curve exhibits the subcritical 

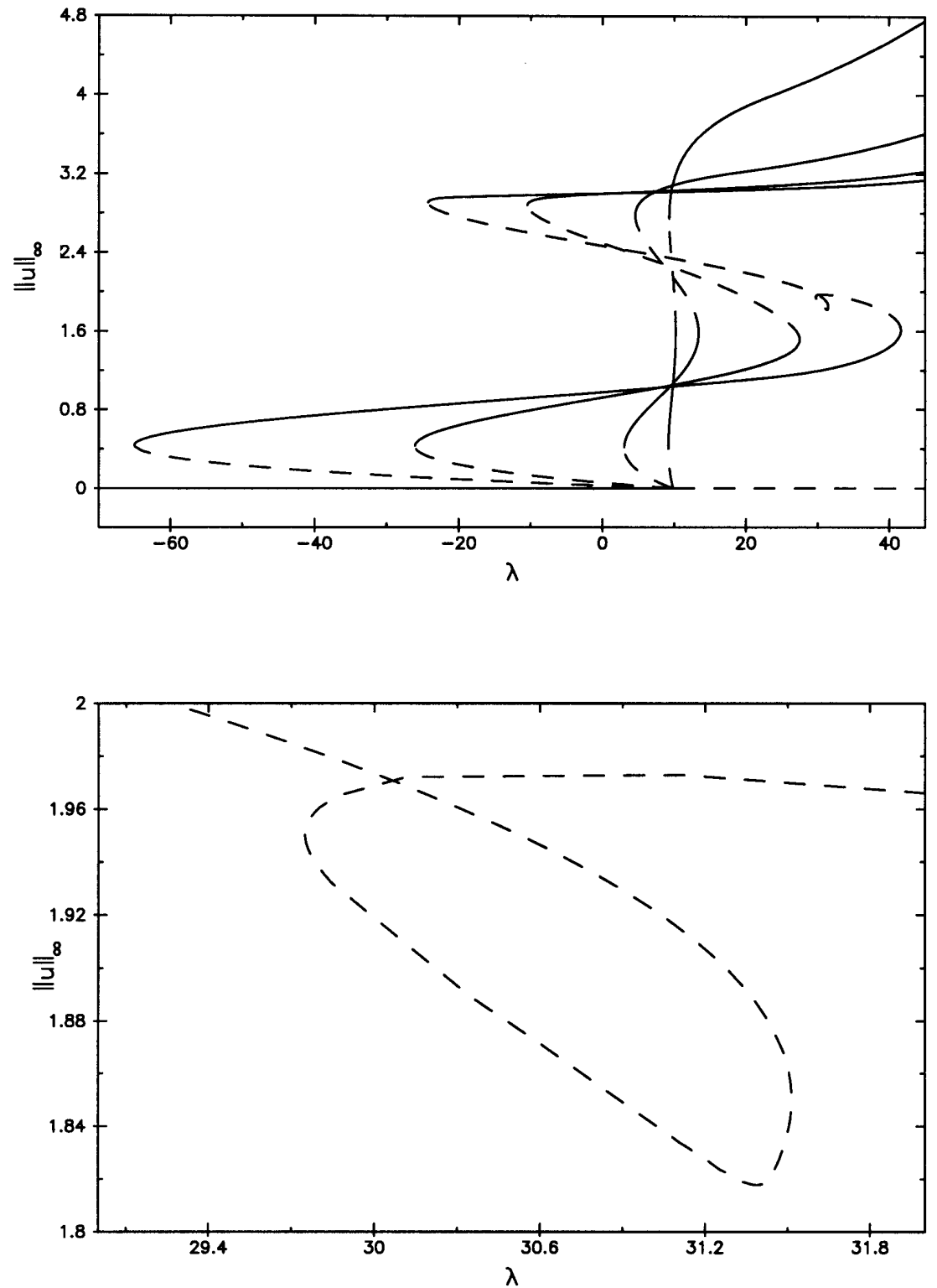

Fig. 5.1. Variation of the bifurcation diagram as $\varepsilon$ grows.

turning point. In this case the problem admits at least three solutions for each $\lambda \in\left(\pi^{2}, 10.2233\right)$. When $\varepsilon=10$, the subcritical turning point of the curve moves up to 13.4255 and hence, for each $\lambda \in\left(\pi^{2}, 13.4255\right)$ the problem possesses at least three solutions. When $\varepsilon=50$ the subcritical turning point grows up to 27.5376 and so the problem possesses at least three solutions for each $\lambda \in\left(\pi^{2}, 27.5376\right)$. 
Finally, if $\varepsilon=100$ then the numerical computations show that (1.1) with the choice (5.1) possesses at least three positive solutions for each $\lambda \in\left(\pi^{2}, 41.6149\right)$, but in this case a further feature arises: The arc of the curve after the subcritical turning point exhibits a loop which did not appear for the previous values of $\varepsilon$. This loop has been magnified in the second picture of Figure 5.1. The delimiting turning points of this loop are located at $\lambda=29.7489$ and $\lambda=31.5152$. Therefore, if $\varepsilon=100$, then the problem possesses at least five positive solutions for each $\lambda \in(29.7489,31.5152)$. In each of these examples the numerical computations suggest that the problem possesses a unique positive solution for $\lambda$ greater than the value of $\lambda$ where the subcritical turning point arises. Since the existence of isolas was not excluded, we cannot be sure if for these ranges of $\lambda$ the problem possesses a unique solution or not. Nevertheless, if the existence of isolas is excluded, then the interval of $\lambda$ 's where uniqueness occurs decreases as $\varepsilon$ grows.

Now, instead of the amplitude of the weight function, we change the thickness of the support of $a(x)$. For this, we consider (1.1) with

$$
\Omega=(0,1), \quad r=4, \quad g(x, u)=11 u^{2}-6 u^{3}-6 u,
$$

and $a \in\left\{a_{1}, \ldots, a_{4}\right\}$, where

$$
\begin{aligned}
& a_{1}(x):=\max \left\{0,-100 x^{2}+100 x-24\right\}, \\
& a_{2}(x):=\max \left\{0, \frac{4}{9}\left(-25 x^{2}+25 x-4\right)\right\}, \\
& a_{3}(x):=\max \left\{0, \frac{1}{4}\left(-25 x^{2}+25 x-\frac{9}{4}\right)\right\}, \\
& a_{4}(x):=\max \left\{0, \frac{1}{144}\left(-625 x^{2}+625 x-\frac{49}{4}\right)\right\} .
\end{aligned}
$$

All these weights have amplitude 1, each of them being supported in

$$
(0.4,0.6), \quad(0.2,0.8), \quad(0.1,0.9), \quad(0.02,0.98),
$$

respectively, and so their respective thicknesses are $T_{1}:=0.2, T_{2}:=0.6, T_{3}:=0.8$ and $T_{4}:=0.96$. The corresponding bifurcation diagrams look like those shown in Figure 5.1, but now the subcritical turning points are located at $\lambda_{1}=10.0166$, $\lambda_{2}:=10.2233, \lambda_{3}:=10.2509$ and $\lambda_{4}=10.2540$, respectively. Thus, though these values increase with the thickness, their variations are far from significant. Moreover, increasing thickness makes $\Omega_{0}$ smaller and hence, the points where bifurcation to infinity occurs grow from 61.6850 up to 24674.0110 . Therefore, the numerics suggest that in practice the interval where uniqueness occurs enlarges when the thickness increases. We conjecture that this is the case. Unfortunately, Theorem 4.1 does not provide us with a proof of this conjecture.

\section{Convergence of the Positive Solutions in $D$}

In this section we complete the analysis of Section 3 ascertaining the pointwise growth as $\lambda \uparrow \sigma_{1}^{\Omega_{0}}[-\Delta]$ of an arbitrary family $\left\{u_{\lambda}\right\}, \sigma_{1}^{\Omega}[-\Delta]<\lambda<\sigma_{1}^{\Omega_{0}}[-\Delta]$, of positive solutions of (1.1). First we analyze the pure logistic problem $(g=0)$. Then, we consider the general problem (1.1). 


\subsection{The case $g=0$}

In case $g=0$, the following result characterizes the limiting behavior in $D$ as $\lambda \uparrow \sigma_{1}^{\Omega_{0}}[-\Delta]$ of the positive solutions $\theta_{[\lambda, a, 0]}$ of (1.1).

Theorem 6.1. Assume that $g=0$ and $a(x)$ satisfies $(\mathrm{H} a)$. Then (i) For each $x \in D$ the pointwise limit

$$
\bar{u}(x):=\lim _{\lambda \uparrow \sigma_{1}^{\Omega_{0}}[-\Delta]} \theta_{[\lambda, a, 0]}(x)<\infty
$$

is well defined.

(ii) $\bar{u} \in W_{\mathrm{loc}}^{2, p}(D) \cap C^{1, v}(D)$ for all $p>1$ and $v \in(0,1)$ and $\bar{u}$ is a weak solution of the equation

$$
-\Delta u=\lambda u-a(x) u^{r+1}
$$

in the domain D. Moreover,

$$
\lim _{\lambda \uparrow \sigma_{1}^{\Omega_{0}[-\Delta]}}\left\|\theta_{[\lambda, a, 0]}-\bar{u}\right\|_{C^{1, v}(D)}=0 .
$$

In the proof of this result we need the following radial counterpart of Lemma 4.3 .

Lemma 6.2. Let $B \subset \mathbb{R}^{N}$ denote the ball of radius $R$ centered at 0 and consider the boundary-value problem

$$
-\Delta u=\lambda u-A u^{r+1} \quad \text { in } B,\left.\quad u\right|_{\partial B}=\sigma,
$$

where $\lambda>0, \sigma>(\lambda / A)^{1 / r}, r>0$ and $A>0$ are constants. Then, (6.1) possesses a unique positive solution $u_{\sigma} \in C^{\infty}(\bar{B})$, the mapping $\sigma \rightarrow u_{\sigma}$ is pointwise increasing in $B$ and

$$
u_{\infty}(x):=\lim _{\sigma \uparrow \infty} u_{\sigma}(x)=\sup _{\sigma>0} u_{\sigma}(x)<\infty \quad \forall x \in B .
$$

Moreover, $u_{\infty}$ provides the minimal classical solution to the singular boundaryvalue problem

$$
-\Delta u=\lambda u-A u^{r+1} \quad \text { in } B,\left.\quad u\right|_{\partial B}=\infty,
$$

where the boundary condition must be understood in the sense that $u(x) \uparrow \infty$ as $\operatorname{dist}(x, \partial B) \downarrow 0$.

Proof. The proof of the existence and the uniqueness of a positive solution for (4.5) applies mutatis mutandis to (6.1). So, (6.1) possesses a unique positive solution. Let $u_{\sigma}$ denote it. Since $(\lambda / A)^{1 / r}$ is a subsolution of (6.1) and $\sigma$ is a supersolution of (6.1), the uniqueness of the positive solution together with the strong maximum principle provide us with the estimate

$$
(\lambda / A)^{1 / r}<u_{\sigma}<\sigma \text { in } B .
$$


A further bootstrapping argument together with the $L^{p}$-estimates of AGMON, Douglis \& Nirenberg and Schauder's theory give $u_{\sigma} \in C^{\infty}(\bar{B})$ (cf. [10, Chap. VI, IX]). Moreover, by the uniqueness of the positive solution, $u_{\sigma}$ must be radially symmetric, since the Laplacian is invariant by rotations.

Hence, there exists $U_{\sigma} \in C^{\infty}([0, R])$ such that $u_{\sigma}(x)=U_{\sigma}(s), s=|x|$. The function $U_{\sigma}$ is the unique positive solution of the one-dimensional problem

$$
\begin{gathered}
-\frac{d}{d s}\left(s^{N-1} \frac{d U_{\sigma}}{d s}\right)=s^{N-1}\left(\lambda U_{\sigma}-A U_{\sigma}^{r+1}\right) \quad 0<s<R, \\
\frac{d U_{\sigma}}{d s}(0)=0, \quad U_{\sigma}(R)=\sigma .
\end{gathered}
$$

Now, the change of variable

$$
\rho=g(s):=\left\{\begin{array}{cl}
\frac{1}{N-2}\left[\frac{1}{s^{N-2}}-\frac{1}{R^{N-2}}\right], & N \geqq 3, \\
\log \left(\frac{R}{s}\right), & N=2,
\end{array} \quad v_{\sigma}(\rho)=U_{\sigma}\left(g^{-1}(\rho)\right),\right.
$$

transforms (6.4) into

$$
\begin{gathered}
-v_{\sigma}^{\prime \prime}=\left(g^{-1}(\rho)\right)^{2(N-1)}\left(\lambda v_{\sigma}-A v_{\sigma}^{r+1}\right), \quad \rho>0 \\
v_{\sigma}(0)=\sigma, \quad v_{\sigma}^{\prime}(\infty)=0
\end{gathered}
$$

where' stands for derivation with respect to $\rho$. By $(6.3), v_{\sigma}>(\lambda / A)^{1 / r}$ and hence,

$$
\lambda v_{\sigma}-A v_{\sigma}^{r+1}<0
$$

Thus, $v^{\prime}(\rho)<0$ for each $\rho>0$. Now, fix $\rho_{1}>0$. Since $g$ is decreasing, for $0<\rho<\rho_{1}$, we have $g^{-1}(\rho)>g^{-1}\left(\rho_{1}\right)$ and hence

$$
-v_{\sigma}^{\prime \prime}=\left(g^{-1}(\rho)\right)^{2(N-1)}\left(\lambda v_{\sigma}-A v_{\sigma}^{r+1}\right)<\left(g^{-1}\left(\rho_{1}\right)\right)^{2(N-1)}\left(\lambda v_{\sigma}-A v_{\sigma}^{r+1}\right) .
$$

Thus, $v_{\sigma}$ is a subsolution of the problem

$$
\begin{gathered}
-w^{\prime \prime}=\left(g^{-1}\left(\rho_{1}\right)\right)^{2(N-1)}\left(\lambda w-A w^{r+1}\right) \quad 0<\rho<\rho_{1}, \\
w(0)=w\left(\rho_{1}\right)=\sigma .
\end{gathered}
$$

Thanks to Lemma $4.3,(6.6)$ possesses a unique positive solution. Let $w_{\sigma}$ denote it. By the uniqueness of the positive solution, it follows from the strong maximum principle that

$$
v_{\sigma}(\rho)<w_{\sigma}(\rho) \quad \forall \rho \in\left(0, \rho_{1}\right]
$$

where we have used that $v_{\sigma}\left(\rho_{1}\right)<\sigma$. Hence, for each $\rho \geqq \frac{1}{2} \rho_{1}$ we find that

$$
v_{\sigma}(\rho) \leqq v_{\sigma}\left(\frac{1}{2} \rho_{1}\right) \leqq w_{\sigma}\left(\frac{1}{2} \rho_{1}\right)=\min _{\left(0, \rho_{1}\right)} w_{\sigma}:=w_{0}(\sigma)<\lim _{\sigma \rightarrow \infty} w_{0}(\sigma):=w_{0}(\infty) .
$$


Moreover, thanks to Lemma 4.3, the following estimate for $w_{0}(\infty)$ holds:

$$
w_{0}(\infty) \leqq \frac{\lambda(r+2)}{2 A}+\frac{2 B_{0}^{2}}{(r+2) A \rho_{1}^{2}\left(g^{-1}\left(\rho_{1}\right)\right)^{2(N-1)}} .
$$

Therefore, since $\rho_{1}>0$ is arbitrary,

$$
u_{\infty}(x):=\lim _{\sigma \rightarrow \infty} u_{\sigma}(x)=\sup _{\sigma \rightarrow \infty} u_{\sigma}(x)<\infty \quad \text { for each } x \in B .
$$

Moreover, (6.7) implies that

$$
u_{\infty}(x) \leqq \frac{\lambda(r+2)}{2 A}+\frac{2 B_{0}^{2}}{(r+2) A \rho_{1}^{2}\left(g^{-1}\left(\rho_{1}\right)\right)^{2(N-1)}}, \quad \rho_{1}=2 \rho(|x|) .
$$

We now show that $u_{\infty}$ is a solution of $-\Delta u=\lambda u-A u^{r+1}$ in $B$. Set

$$
U_{0, \sigma}:=\inf _{(0, R)} U_{\sigma}=U_{\sigma}(0), \quad U_{0, \infty}:=U_{\infty}(0),
$$

where $U_{\infty}$ is the unique function such that $u_{\infty}(x)=U_{\infty}(s), s=|x|$. Since $U_{\sigma}$ satisfies (6.4),

$$
U_{\sigma}(s)=U_{0, \sigma}-\int_{0}^{s} \int_{0}^{\rho}\left(\frac{\tau}{\rho}\right)^{N-1}\left(\lambda U_{\sigma}-A U_{\sigma}^{r+1}\right) d \tau d \rho
$$

for each $s<R$, and hence, passing to the limit as $\sigma \uparrow \infty$ in (6.8), we find from the theorem of dominated convergence that

$$
U_{\infty}(s)=U_{0, \infty}-\int_{0}^{s} \int_{0}^{\rho}\left(\frac{\tau}{\rho}\right)^{N-1}\left(\lambda U_{\infty}-A U_{\infty}^{r+1}\right) d \tau d \rho .
$$

Since (6.9) holds for each $s<R$, it readily follows that $U_{\infty} \in C^{\infty}(B)$. Moreover, by the uniqueness of the solution of the Cauchy problem, $U_{\infty}(s)$ is the unique positive solution of

$$
\begin{gathered}
-\left(s^{N-1} u^{\prime}\right)^{\prime}=s^{N-1}\left(\lambda u-A u^{r+1}\right), \quad 0<s<R, \\
u(0)=U_{0, \infty}, \quad u^{\prime}(0)=0,
\end{gathered}
$$

which is defined on $0 \leqq s<R$. Since $U_{\infty}(s)$ is increasing and $\lim _{s \uparrow R} U_{\infty}(s)=\infty$, we are done.

To complete the proof it remains to show the minimal character of $U_{\infty}$ among all positive solutions. Note that given any positive classical solution $u$ of (6.2) for each $\sigma>0$ there exists $r(\sigma)<R$ such that

$$
u_{\sigma}(x)<u(x), \quad r(\sigma) \leqq|x|<R .
$$

By the uniqueness of the positive solution, the strong maximum principle implies that $u>u_{\sigma}$ in $|x| \leqq r(\sigma)$ as well and hence, $u_{\sigma}<u$. Therefore, passing to the limit as $\sigma \uparrow \infty$ yields $u_{\infty} \leqq u$. This completes the proof. 
Proof of Theorem 6.1. Let $B$ be an arbitrary ball such that $\bar{B} \subset D$ and take $\lambda \in\left(\sigma_{1}^{\Omega}[-\Delta], \sigma_{1}^{\Omega_{0}}[-\Delta]\right)$. Then, the restriction $u:=\left.\theta_{[\lambda, a, 0]}\right|_{B}$ satisfies

$$
-\Delta u=\lambda u-a u^{r+1}<\sigma_{1}^{\Omega_{0}}[-\Delta] u-\left(\inf _{B} a\right) u^{r+1}
$$

and hence,

$$
\theta_{[\lambda, a, 0]}<u_{\infty, B} \text { in } \bar{B},
$$

where $u_{\infty, B}$ stands for the minimal classical solution of

$$
-\Delta u=\sigma_{1}^{\Omega_{0}}[-\Delta] u-\left(\inf _{B} a\right) u^{r+1} \quad \text { in } B,\left.\quad u\right|_{\partial B}=\infty,
$$

whose existence is given by Lemma 6.2. Therefore, since $\theta_{[\lambda, a, 0]}$ is pointwise increasing in $\lambda$, the pointwise limit $\bar{u}(x):=\lim _{\lambda \uparrow \sigma_{1}^{\Omega_{0}}[-\Delta]} \theta_{[\lambda, a, 0]}(x)$ is well defined and finite for each $x \in D$.

Let $B^{\prime}$ and $B^{\prime \prime}$ be two arbitrary concentric balls contained in $B$, with $B^{\prime} \subset$ $\overline{B^{\prime}} \subset B^{\prime \prime} \subset \overline{B^{\prime \prime}} \subset B$. For each $p>1$ we have $\theta_{[\lambda, a, 0]} \in W^{2, p}(B)$. Moreover, $\theta_{[\lambda, a, 0]}<u_{\infty, B}$. Thus, from the interior $L^{p}$-estimates and MORREY's embedding theorem we find that for each $v \in(0,1)$ there exists $C=C\left(\nu, B^{\prime}, B^{\prime \prime}\right)$ such that

$$
\left\|\theta_{[\lambda, a, 0]}\right\|_{C^{1, v}\left(\overline{B^{\prime}}\right)} \leqq C
$$

for any $\lambda \in\left(\sigma_{1}^{\Omega}[-\Delta], \sigma_{1}^{\Omega_{0}}[-\Delta]\right)$. Now, a rather standard compactness argument together with the uniqueness of the pointwise limit $\bar{u}$ shows that

$$
\lim _{\lambda \uparrow \sigma_{1}^{\Omega_{0}}[-\Delta]}\left\|\theta_{[\lambda, a, 0]}-\bar{u}\right\|_{C^{1, v}\left(\bar{B}^{\prime}\right)}=0,
$$

and therefore,

$$
\lim _{\lambda \uparrow \sigma_{1}^{\Omega_{0}[-\Delta]}}\left\|\theta_{[\lambda, a, 0]}-\bar{u}\right\|_{C^{1, v}(D)}=0 .
$$

In particular, $\bar{u}$ must be a weak solution of $-\Delta u=\sigma_{1}^{\Omega_{0}}[-\Delta] u-a u^{r+1}$ in $D$. By elliptic regularity, $\bar{u} \in W_{\text {loc }}^{2, p}(D)$ for each $p>1$. This completes the proof.

If we define a weak solution $u$ of the problem

$$
-\Delta u=\lambda u-a u^{r+1} \text { in } D,\left.\quad u\right|_{\partial D}=\infty,
$$

to be a function $u \in W_{\text {loc }}^{1, p}(D)$, for some $p>1$, such that $u(x) \rightarrow \infty$ as dist $(x, \partial D) \downarrow 0$ (cf. [11], for instance), and we assume that $a(x)$ and $\partial D$ satisfy the assumptions of Corollary 3.3, then $\lim _{\lambda \uparrow \sigma_{1}^{\Omega_{0}}[-\Delta]} \theta_{[\lambda, a, 0]}=\infty$ uniformly on $\partial D$ and therefore, $\bar{u}$ provides us with a weak solution of (6.11). Moreover, the corresponding argument in the proof of Lemma 6.2 can be adapted to show that in fact $\bar{u}$ is the minimal weak solution of (6.11). All these features can be summarized in the following result.

Corollary 6.3. Let $\bar{u}=\lim _{\lambda \uparrow \sigma_{1}^{\Omega_{0}}[-\Delta]} \theta_{[\lambda, a, 0]}$ and suppose that $a(x)$ and $\partial D$ satisfy the assumptions of Corollary 3.3. Then, $\bar{u}$ is the minimal weak solution of (6.11). 


\subsection{The general problem}

Here, we show that Theorem 6.1(i) extends to cover the general case when $g(x, u)$ satisfies $(\mathrm{H} g)$ and the following growth condition at infinity:

$$
g(x, u)=o\left(u^{r-\delta}\right) \quad \text { as } u \rightarrow \infty \quad \text { uniformly in } \Omega,
$$

where $\delta \in(0, r)$. Moreover, if in addition we assume (1.1) to possess a unique positive solution for each $\lambda$ in a left neighborhood of $\sigma_{1}^{\Omega_{0}}[-\Delta]$, then Theorem 6.1(ii) and Corollary 6.3 also hold.

We begin by analyzing the special model (4.20) in case $b>0$. For this model conditions $(\mathrm{Hg})$ and (6.12) are satisfied. Thanks to Theorem 4.4 there exists $\lambda_{0} \in$ $\left(\sigma_{1}^{\Omega}[-\Delta], \sigma_{1}^{\Omega_{0}}[-\Delta]\right)$ such that for each $\lambda \in\left[\lambda_{0}, \sigma_{1}^{\Omega_{0}}[-\Delta]\right)$ the problem (4.20) possesses a unique positive solution. Let us denote it by $u_{\lambda}$. It is easily seen that $\lambda \rightarrow u_{\lambda}$ is pointwise increasing. Moreover, by (4.21) we have that $u_{\lambda_{0}} \geqq b^{1 / \delta}$ on $\bar{D}$. Thus, there exist $c_{0}>b^{1 / \delta}$ and $\lambda_{1} \in\left(\lambda_{0}, \sigma_{1}^{\Omega_{0}}[-\Delta]\right)$ such that

$$
u_{\lambda} \geqq c_{0}>b^{1 / \delta} \quad \text { in } \bar{D}
$$

for all $\lambda \in\left[\lambda_{1}, \sigma_{1}^{\Omega_{0}}[-\Delta]\right)$. Therefore,

$$
\frac{u^{r+1}-b u^{r+1-\delta}}{u^{r+1}} \geqq c_{1}:=1-b c_{0}^{-\delta}, \quad \forall u \geqq c_{0} .
$$

We now show the existence of local uniform estimates in $D$ for the family $u_{\lambda}$, $\lambda \geqq \lambda_{1}$. Let $B$ be an arbitrary ball with $B \subset \bar{B} \subset D$. By (6.13) and (6.14), we have that

$$
-\Delta u_{\lambda} \leqq \sigma_{1}^{\Omega_{0}}[-\Delta] u_{\lambda}-c_{1}\left(\inf _{B} a\right) u_{\lambda}^{r+1} \text { in } B
$$

for each $\lambda \in\left[\lambda_{1}, \sigma_{1}^{\Omega_{0}}[-\Delta]\right)$. Thus,

$$
u_{\lambda} \leqq u_{\infty, B} \quad \text { in } B,
$$

where $u_{\infty, B}$ is the minimal positive solution of

$$
-\Delta u=\sigma_{1}^{\Omega_{0}}[-\Delta] u-c_{1}\left(\inf _{B} a\right) u^{r+1} \quad \text { in } B,\left.\quad u\right|_{\partial B}=\infty,
$$

whose existence follows from Lemma 6.2. Therefore, for each $x \in D$ we find that

$$
\bar{u}(x):=\lim _{\lambda \uparrow \sigma_{1}^{\Omega_{0}}[-\Delta]} u_{\lambda}(x)<\infty .
$$

This shows the validity of Theorem 6.1(i) for (4.20). The remaining assertions of Theorem 6.1 follow easily by using the same argument as in its proof. Therefore, Theorem 6.1 is valid for (4.20).

Now, we consider (1.1) with $g$ satisfying $(\mathrm{H} g)$ and (6.12). Fix $\lambda_{1} \in\left(\sigma_{1}^{\Omega}, \sigma_{1}^{\Omega_{0}}\right)$. By Lemma 3.2 there exists a constant $c=c\left(\lambda_{1}\right)>0$ such that

$$
\inf _{x \in D} u(x) \geqq c>0
$$


for each positive solution of (1.1) with $\lambda \geqq \lambda_{1}$. Moreover, thanks to (6.12), there exists a constant $b_{1}>0$ such that $\frac{|g|}{u^{r-\delta}} \leqq b_{1}$ if $u \geqq c$. Thus, for each $\lambda \geqq \lambda_{1}$ any positive solution $u$ of (1.1) satisfies

$$
-\Delta u \geqq \lambda u-a\left(u^{r+1}-b_{1} u^{r+1-\delta}\right) \quad \text { in } \Omega,
$$

and hence it is a supersolution of

$$
-\Delta v=\lambda v-a\left(v^{r+1}-b_{1} v^{r+1-\delta}\right) \quad \text { in } \Omega,\left.\quad v\right|_{\partial \Omega}=0 .
$$

On the other hand, thanks to Theorem 4.4, there exists $\lambda_{2} \in\left[\lambda_{1}, \sigma_{1}^{\Omega_{0}}[-\Delta]\right)$ such that (6.15) possesses a unique positive solution for each $\lambda \in\left[\lambda_{2}, \sigma_{1}^{\Omega_{0}}[-\Delta]\right)$. Thus, if $u_{\lambda, 1}$ stands for the unique positive solution of (6.15), then $u_{\lambda} \leqq u_{\lambda, 1}$ for any positive solution $u_{\lambda}$ of (1.1) with $\lambda \in\left[\lambda_{2}, \sigma_{1}^{\Omega_{0}}[-\Delta]\right)$. Therefore,

$$
\limsup _{\lambda \uparrow \sigma_{1}^{\Omega_{0}}[-\Delta]} u_{\lambda}(x) \leqq \bar{u}_{1}(x):=\lim _{\lambda \uparrow \sigma_{1}^{\Omega_{0}}[-\Delta]} u_{\lambda, 1}(x)<\infty \quad \forall x \in D .
$$

Moreover, a further compactness argument as in the proof of Theorem 6.1 shows that given any family $\left\{u_{\lambda}\right\}$ of positive solutions of $(1.1), \sigma_{1}^{\Omega}[-\Delta]<\lambda<\sigma_{1}^{\Omega_{0}}[-\Delta]$, there exist a sequence $\lambda_{n} \uparrow \sigma_{1}^{\Omega_{0}}[-\Delta]$ and a weak solution $\bar{u} \in W_{\mathrm{loc}}^{2, p}(D) \cap C^{1, v}(D)$, for each $p>1$ and $0<v<1$, of the equation

$$
-\Delta u=\sigma_{1}^{\Omega_{0}}[-\Delta] u-a\left(u^{r}+g(x, u)\right) u
$$

in the domain $D$ such that

$$
\lim _{n \rightarrow \infty}\left\|u_{\lambda_{n}}-\bar{u}\right\|_{C^{1, v}(D)}=0
$$

for each $v \in(0,1)$. In general, $\bar{u}$ is not uniquely determined. Nevertheless, if (1.1) possesses a unique positive solution for each $\lambda$ in a left neighborhood of $\sigma_{1}^{\Omega_{0}}[-\Delta]$, then $\bar{u}$ is unique and all the conclusions of Theorem 6.1 hold. All these features can be summarized in the following result.

Theorem 6.4. Assume that a $(x)$ satisfies $(\mathrm{H} a)$ and that $g(x, u)$ satisfies $(\mathrm{H} g \mathrm{w})$ and (6.12). Then (i) Any family $\left\{u_{\lambda}\right\}$ of positive solutions to (1.1), $\lambda \in\left(\sigma_{1}^{\Omega}, \sigma_{1}^{\Omega_{0}}\right)$, is uniformly bounded above in $D$, with the upper bound independent of the family.

(ii) Let $\left\{u_{\lambda}\right\}$ be any family as in (i) and consider any sequence $\Lambda=\left\{\lambda_{n}\right\}$, such that $\lim _{n \rightarrow \infty} \lambda_{n}=\sigma_{1}^{\Omega_{0}}[-\Delta]$. Then, there exist a subsequence of $\Lambda, \hat{\Lambda}:=\left\{\hat{\lambda}_{n}\right\}$, and a weak solution $\bar{u}_{\hat{\Lambda}}$ of (6.16) in $D, \bar{u}_{\hat{\Lambda}} \in W_{\mathrm{loc}}^{2, p}(D) \cap C^{1, v}(D)$, for each $p>1$, $0<v<1$, such that

$$
\lim _{n \rightarrow \infty}\left\|u_{\hat{\lambda}_{n}}-\bar{u}_{\hat{\Lambda}}\right\|_{C^{1, v}(D)}=0
$$

for each $v \in(0,1)$.

(iii) If (1.1) possesses a unique positive solution for each $\lambda$ in a left neighborhood of $\sigma_{1}^{\Omega_{0}}[-\Delta]$ denoted by $u_{\lambda}$, then the function $\bar{u}$ given by

$$
\bar{u}(x):=\lim _{\lambda \uparrow \sigma_{1}^{\Omega_{0}}[-\Delta]} u_{\lambda}(x) \quad \forall x \in D
$$


is well defined and provides a weak solution of (6.16) in $W_{\mathrm{loc}}^{2, p}(D) \cap C^{1, v}(D)$, for each $p>1$ and $0<v<1$. Moreover,

$$
\lim _{\lambda \uparrow \sigma_{1}^{\Omega_{0}[-\Delta]}}\left\|u_{\lambda}-\bar{u}\right\|_{C^{1, v}(D)}=0
$$

for each $v \in(0,1)$.

(iv) Under the assumptions of Corollary 3.3, each of the functions $\bar{u}_{\hat{\Lambda}}$ of part (ii) and $\bar{u}$ of part (iv), defines a weak solution to the singular boundary-value problem

$$
-\Delta u=\lambda u-a\left(u^{r}+g(x, u)\right) u \quad \text { in } D,\left.\quad u\right|_{\partial D}=\infty .
$$

Moreover, in the context of (iii), $\bar{u}$ is the minimal weak solution of (6.18).

Remark 6.5. The growth condition (6.12) can be slightly weakened by simply assuming that $g^{-}=o\left(u^{r-\delta}\right)$ as $u \uparrow \infty$, for some $0<\delta<r$, where $g^{-}=\min \{g, 0\}$.

Acknowledgements. The numerical calculations were done by adapting a path-following scheme by Professor M. MOLINA-MEYER at Carlos III University. We sincerely thank her for the original scheme and her invaluable help. The research for this work was supported by the Spanish DGICYT and DGES under grants PB93-0465 and PB96-0621.

\section{References}

1. H. Amann, Fixed point equations and nonlinear eigenvalue problems in ordered Banach spaces, SIAM Review 18 (1976) 620-709.

2. R. A. Adams Sobolev Spaces, Academic Press, New York, 1975.

3. H. Brezis \& L. Oswald, Remarks on sublinear elliptic equations, Nonl. Anal. TMA 10 (1986) 55-64.

4. R. Courant \& D. Hilbert, Methods of Mathematical Physics, Vol. I Wiley, New York, 1962

5. M. G. Crandall \& P. H. Rabinowitz, Bifurcation from simple eigenvalues, J. Funct. Anal. 8 (1971) 321-340.

6. M. G. Crandall \& P. H. Rabinowitz, Bifurcation, perturbation of simple eigenvalues and linearized stability, Arch. Rational Mech. Anal. 52 (1973) 161-180.

7. J. M. Fraile, P. Koch-Medina, J. López-Gómez \& S. Merino, Elliptic eigenvalue problems and unbounded continua of positive solutions of a semilinear equation, J. Diff. Eqns. 127 (1996) 295-319.

8. J. M. Fraile, J. LóPez-Gómez \& J. C. Sabina, On the global structure of the set of positive solutions of some semilinear elliptic boundary value problems, J. Diff. Eqns. 123 (1995) 180-212.

9. J. L. GÁMEZ, Sub- and Super-solutions in bifurcation problems, Nonl. Anal. TMA 28 (1997) 625-632.

10. D. Gilbarg \& N. Trudinger, Elliptic Partial Differential Equations of Second Order, Springer-Verlag, Berlin, New York, 1983.

11. A. C. LAzER \& P. J. MCKenNA, A singular elliptic boundary value problem, Appl. Maths. Comp. 65 (1994) 183-194.

12. J. LÓPEZ-GóMEZ, The maximum principle and the existence of principal eigenvalues for some linear weighted boundary value problems, J. Diff. Eqns. 127 (1986) 263-294.

13. J. LóPEZ-GómEz, On the uniqueness of positive solutions for a class of sublinear elliptic problems, Boll. Un. Mat. Ital. 11-B (1997) 697-711. 
14. J. López-Gómez, J. C. Eilbeck, K. Duncan \& M. Molina-Meyer, Structure and numerical continuation of solution manifolds in a strong coupled elliptic system, IMA J. Numer. Anal. 12 (1992) 405-428.

15. J. LÓPEZ-Gómez \& J. C. SABINA, First variations of principal eigenvalues with respect to the domain and pointwise growth of positive solutions for problems where bifurcation from infinity occurs, J. Diff. Eqns. 148 (1998) 47-64.

16. J. D. MurRAY, Mathematical Biology, Springer-Verlag, 1993.

17. T. OUYANG, On the positive solutions of semilinear equations $\Delta u+\lambda u-h u^{p}=0$ on the compact manifolds, Trans. Amer. Math. Soc. 331 (1992) 503-527.

18. P. H. RABINOWITZ, Some global results for nonlinear eigenvalue problems, J. Funct. Anal. 7 (1971) 487-513.

19. E. M. Stein, Singular Integrals and Differentiability Properties of Functions, Princeton University Press, Princeton, 1970.

20. J. L. VÁZQUEZ, A strong maximum principle for some quasilinear elliptic equations, Appl. Math. Optim. 12 (1984) 191-202.

J. García, R. Gómez, J. C. SABina de Lis Departamento de Análisis Matemático Universidad de La Laguna 38271-La Laguna (Tenerife), Spain

and

J. LÓPEZ-GÓMEZ

Departamento de Matemática Aplicada

Universidad Complutense de Madrid 28040-Madrid, Spain 